\title{
Fully three-dimensional analysis of high-speed train-track-soil-structure dynamic interaction
}

\author{
P. Galvín, A. Romero, J. Domínguez \\ Escuela Técnica Superior de Ingenieros, Universidad de Sevilla, Camino de los Descubrimientos, 41092 Sevilla, Spain
}

\begin{abstract}
In this paper, a general and fully three dimensional multi-body-finite element-boundary element model, formulated in the time domain to predict vibrations due to train passage at the vehicle, the track and the free field, is presented. The vehicle is modelled as a multi-body system and, therefore, the quasi-static and the dynamic excitation mechanisms due to train passage can be considered. The track is modelled using finite elements. The soil is considered as a homogeneous half-space by the boundary element method. This methodology could be used to take into account local soil discontinuities, underground constructions such as underpasses, and coupling with nearby structures that break the uniformity of the geometry along the track line. The non-linear behaviour of the structures could be also considered. In the present paper, in order to test the model, vibrations induced by high-speed train passage are evaluated for a ballasted track. The quasi-static and dynamic load components are studied and the influence of the suspended mass on the vertical loads is analyzed. The numerical model is validated by comparison with experimental records from two HST lines. Finally, the dynamic behaviour of a transition zone between a ballast track and a slab track is analyzed and the obtained results from the proposed model are compared with those obtained from a model with invariant geometry with respect to the track direction.
\end{abstract}

Key words: HST vibrations, vehicle-track-soil-structure interaction, Dynamic loads, BEM/FEM coupling

\section{Introduction}

The emergence of high-speed trains (HST) in Spain has been decisive for its economic and social regional development. The Spanish Government is developing the Infrastructures and Transports Strategic Plan where, the National Administrator of Railways (ADIF) is committed to construct HST lines to reach a total of $10000 \mathrm{~km}$ before 2020. At present, there are $1.594 \mathrm{~km}$ HST lines in operation, $2.214 \mathrm{~km}$ under construction, and $1.720 \mathrm{~km}$ have already been planned [1].

The construction of many HST lines in Spain, other European countries and Asia has pushed the railway engineering community to conduct much research related to different aspects of HST passage effects in recent

Email address: pedrogalvin@us.es (P. Galvín)

Preprint submitted to Journal of Sound and Vibration

June 14, 2010 
years. Vehicle, track, soil, and structure dynamic behaviours have been studied, leading to the conclusion that dynamic effects are much more important for HST than for conventional trains [2]. These effects require a deep analysis in order to maintain security and comfort in the trains and to avoid problems due to vibrations induced in nearby constructions by waves transmitted through the soil.

One of the first steps in the study of vibrations induced by HST is an accurate modelling of the force induced by the train which is transmitted to the soil through the track. This force is generated by a variety of excitation mechanisms: the quasi-static contribution (force generated by moving axle loads), the parametric excitation due to discrete supports of the rails, the transient excitation due to rail joints and wheel flats, and the excitation due to wheel and rail roughness and track unevenness [3]. In the early studies, a prediction model developed by Krylov $\underline{[4]}$ was used $\underline{[5,} \underline{6]}$. In that model, only the quasi-static force transmitted by the sleepers in a ballasted track is taken into account as a moving force. Krylov's model is valid when the train speed is close to the critical phase velocity of the coupled track-soil system because in this case the quasi-static excitation is dominant $\underline{\underline{77}} \underline{\underline{8}, \underline{9]}}$.

Recently, more advanced models have been used allowing the dynamic train-track interaction mechanisms and the actual track properties to be considered. Lombaert et al. $\underline{[8,} \underline{10]}$ have applied the formulation presented by Metrikine et al. [11], Metrikine and Popp [12], and Dieterman and Metrikine [13], to predict vibrations produced by railway traffic using a coupled two-and-half boundary element-finite element formulation in the frequency domain. In that approach the vehicle is coupled to an infinite length beam that represents the track and a half-space representing the soil. The model has been validated by experimental results $\underline{[8,10]}$. Auersch $\underline{[9,} \underline{14]}$ has presented a model for ground-borne railway induced vibrations formulated in a similar way. Takemiya and Bian $\underline{[15]}$ have extended the model presented by Metrikine and Popp $\underline{[16]}$ to consider the facts that rails are based discretely on the ground and that sleepers have a significant inertia effect on the rails. They included a layered soil in the analysis and used a discrete Kelvin model for the sleepers' complex frequency-dependent stiffness. Sheng et al. $\underline{[7, \underline{17}} \underline{\underline{18]}}$ also applied a two-and-a-halfdimensional frequency domain model to study vibrations due to train passage. In that model, the dynamic train-track interaction is taken into account using an infinite layered beam for the track coupled to a layered half-space. Xia et al. [19] have presented an integrated train-track-subsoil dynamic interaction model for moving-train induced ground vibration based on vehicle dynamics, track dynamics and Green's functions of the soil.

The previous numerical models, except reference [19], are formulated in the two-and-a-half domain, therefore, it is supposed that the ground and structures such as tunnels and tracks, are homogeneous in the track direction. The models use Green's functions for a layered full-space or for a layered half-space to represent the soil. The objective of the present paper is to develop a general and fully coupled three dimensional multi-body-finite element-boundary element model formulated in the time domain to predict vibrations due to train passage at the vehicle, the track, the free field and structures situated near the track 
(figure 1). The vehicle is modelled as a multi-body system and, therefore, the quasi-static and the dynamic excitation mechanism can be considered. The model has been developed to account for the full dynamic vehicle-track-soil interaction. As compared to two-and-a-half domain solutions, the formulation presented in this paper could be used to take into account local soil discontinuities, underground constructions such as underpasses, and coupling with nearby structures that break the uniformity of the geometry along the track line. However, the main disadvantage of the three dimensional models are that they are computationally expensive. In this model, the track and other structures are modelled using finite elements and their nonlinear behaviour could be also considered since a time domain formulation is employed $\underline{[20]}$. The soil is represented using the boundary element method, in combination with a full-space fundamental solution and quadratic elements [21]. Special attention is paid to stabilization algorithms and element subdivision to improve efficiency, stability and accuracy. Internal material damping is introduced in the boundary element time domain formulation in a simple and efficient manner $[6,22,23]$.

[Figure 1 about here.]

The outline of this paper is as follows. First, the numerical model is presented. This presentation includes: a brief summary of the finite element and the boundary element time domain formulations, and the multibody model used to represent the train-track-soil dynamic interaction. Second, the quasi-static and dynamic load components are studied. The dynamic load is due to rail and wheel irregularities and the parametric excitation is due to the discrete rail support. The influence of the suspended mass on the vertical loads is analyzed. Third, the numerical model is experimentally validated by comparison with existing experimental records taken from two HST lines. Finally, the dynamic behaviour of a transition zone between a ballast track and a slab track is studied. Induced vibrations during HST passage on a transition zone are computed and they are compared with results obtained considering an invariant geometry model with respect to the track direction. A parametric analysis is conducted considering different rail pads and soil properties.

\section{Numerical model}

The model is based on three-dimensional finite element $\underline{[20]}$ and boundary element $\underline{[21]}$ time domain formulations. A direct procedure is used to couple boundary and finite element methods $\underline{[24,} \underline{25,} \underline{\underline{26}}$. A multi-body model is considered to represent the vehicle and to consider the quasi-static and dynamic load components transmitted by the train to the soil through the track.

\subsection{Boundary Element Formulation}

The boundary element method system of equations can be solved step-by-step to obtain the time variation of the boundary unknowns; i.e. displacements and tractions. Piecewise constant time interpolation functions 
are used for tractions and piecewise linear functions for displacements, the fundamental solution displacement and traction are evaluated analytically without much difficulty, and nine node rectangular and six node triangular quadratic elements are used for spatial discretization. Explicit expressions of the fundamental solution displacements and tractions corresponding to an impulse point load in a three dimensional elastic full-space can be seen in reference $\underline{[22]}$. An approach based on the idea of using a linear combination of equations for several time steps in order to advance one step is used to ensure that the stepping procedure is stable in time. Details of this stabilization approach can be found in [27]. Finally, an approach based on the classical Barkan expression [28] is employed to account for material damping in the soil.

Once the integral equation is discretized one obtains the following equation for each time step:

$$
\mathbf{H}^{n n} \mathbf{u}^{n}=\mathbf{G}^{n n} \mathbf{p}^{n}+\sum_{m=1}^{n-1}\left(\mathbf{G}^{n m} \mathbf{p}^{m}-\mathbf{H}^{n m} \mathbf{u}^{m}\right) \exp [-2 \pi \alpha(n-m) \Delta t]
$$

where, $\mathbf{u}^{n}$ is the displacement vector and $\mathbf{p}^{n}$ is the traction vector at the end of the time interval $n$, and $\mathbf{H}^{n n}$ and $\mathbf{G}^{n n}$ are the full unsymmetrical boundary element system matrices, in the time interval $n, \alpha$ is the soil attenuation coefficient and $\Delta t$ is the time step. The right hand side term derived from previous steps is damped by an exponential coefficient using a linearly increasing exponent, with time.

\subsection{Finite Element Formulation}

The equation which results from the finite element method can be expressed symbolically as follows if an implicit time integration Newmark method is applied [29]:

$$
\mathbf{D}^{n n} \mathbf{u}^{n}=\mathbf{f}^{n}+\mathbf{f}^{n-1}
$$

where $\mathbf{D}^{n n}$ is the dynamic stiffness matrix, $\mathbf{u}^{n}$ the displacement vector and $\mathbf{f}^{n}$ the equivalent force vector, in the time interval $n$. Spring-damper, beam, shell and solid elements are used for the discretization.

\subsection{Vehicle model}

The articulated HST studied in this paper has two traction cars and eight passenger cars. It includes a laboratory wagon next to a traction car for the Córdoba-Málaga HST. The two passenger cars adjacent to the traction cars or to the laboratory wagon, share one bogie with the neighbouring passenger car, while the six central passenger cars share both bogies with the neighbouring cars.

[Figure 2 about here.]

The multi-body model shown in figure 3 is used to represent the train-track dynamic interaction due to an axle passage. The primary and secondary suspensions isolate the carriages from the track vibrations. The axles and the car body are considered as rigid parts and the primary and secondary suspensions are represented by spring and damper elements [17]. The coupling between axles on the same bogie, known as leakage, is ignored since not coupling is expected $[6,8,10]$. 
[Figure 3 about here.]

The equations of motion can be written as follows:

$$
\begin{aligned}
& {\left[\begin{array}{cccc}
\bar{M}_{c} & 0 & 0 & 0 \\
0 & \bar{M}_{b} & 0 & 0 \\
0 & 0 & \bar{M}_{w} & 0 \\
0 & 0 & 0 & 0
\end{array}\right]\left[\begin{array}{c}
\ddot{x}_{c} \\
\ddot{x}_{b} \\
\ddot{x}_{w} \\
\ddot{u}_{c}
\end{array}\right]+\left[\begin{array}{cccc}
\bar{c}_{2} & -\bar{c}_{2} & 0 & 0 \\
-\bar{c}_{2} & \bar{c}_{1}+\bar{c}_{2} & -\bar{c}_{1} & 0 \\
0 & -\bar{c}_{1} & \bar{c}_{1} & 0 \\
0 & 0 & 0 & 0
\end{array}\right]\left[\begin{array}{c}
\dot{x}_{c} \\
\dot{x}_{b} \\
\dot{x}_{w} \\
\dot{u}_{c}
\end{array}\right]+} \\
& {\left[\begin{array}{cccc}
\bar{k}_{2} & -\bar{k}_{2} & 0 & 0 \\
-\bar{k}_{2} & \bar{k}_{1}+\bar{k}_{2} & -\bar{k}_{1} & 0 \\
0 & -\bar{k}_{1} & \bar{k}_{1}+\bar{k}_{H} & -\bar{k}_{H} \\
0 & 0 & -\bar{k}_{H} & \bar{k}_{H}
\end{array}\right]\left[\begin{array}{c}
x_{c} \\
x_{b} \\
x_{w} \\
u_{c}
\end{array}\right]=\left[\begin{array}{c}
\bar{M}_{b} \\
\bar{M}_{a} \\
\bar{M}_{w} \\
0
\end{array}\right] \mathbf{g}+\left[\begin{array}{c}
0 \\
0 \\
0 \\
F_{c}
\end{array}\right]}
\end{aligned}
$$

where $x_{c}, x_{b}$ and $x_{w}$ represent the car body, bogie and wheel displacements, respectively. $\mathbf{g}$ is the acceleration due to gravity. The load transmitted by an axle depends on the car body mass, $M_{c}$, the bogie mass, $M_{b}$, the mass of the wheelset, $\bar{M}_{w}$, the primary suspension, $k_{1}$ and $c_{1}$, the secondary suspension, $k_{2}$ and $c_{2}$, and the train configuration. The bogies are supported by two axles, therefore an axle transmits a half of the total bogie mass, $\left(\bar{M}_{b}=M_{b} / 2\right)$. The primary suspension connects the wheels to the bogie, therefore, $\bar{k}_{1}$ and $\bar{c}_{1}$ represent two times the total primary vertical stiffness and the total primary vertical viscous damping, respectively. In a traction car, the car body is supported by two bogies (four axles) and every axle transmits a quarter of the car body mass $\left(\bar{M}_{c}=M_{c} / 4\right)$. The secondary suspension connects the bogie centre to the car body. The car body mass transmitted by an axle is isolated by a half of the secondary suspension. Then, $\bar{k}_{2}$ and $\bar{c}_{2}$ represent a half of the secondary vertical stiffness and secondary vertical viscous damping, respectively. Similar models can be derived for the passenger cars, taking into account the fact that adjacent cars are coupled by shared bogies. $u_{c}$ denotes the total displacement of the rail and $F_{c}$ the contact force between the axle and the rail. Wheel-rail coupling is illustrated in figure 3, where a Hertzian contact spring is inserted between both elements $\left[17, \underline{30]}\right.$. The stiffness of this Hertzian contact spring is denoted by $\bar{k}_{H}$ and its value $2.8 \times 10^{9} \mathrm{~N} / \mathrm{m}$ is taken from $\underline{[30]}$.

The displacement vector $u_{c}$ is equal to the sum of the actual rail displacement $u_{r}$ and the rail unevenness $u_{w / r}$ perceived by an axle $[8, \underline{10]}$ :

$$
u_{c}=u_{r}+u_{w / r}
$$

In this paper, random track unevenness $u_{w / r}(x)$ is modelled as a stationary Gaussian random process characterized by its one-sided PSD function $\tilde{S}_{u_{w / r}}\left(k_{y}\right)$. The spectral representation theorem is used to generate samples of track unevenness $u_{w / r}(x)$ as a superposition of harmonic functions with random phase angles $\underline{[8,} \underline{10]}$ :

$$
u_{w / r}(x)=\sum_{m=1}^{n} \sqrt{2 \tilde{S}_{u_{w / r}}\left(k_{y m}\right) \Delta k_{y}} \cos \left(k_{y m} y-\theta_{m}\right)
$$


where $k_{y m}=m \Delta k_{y}$ is the wavenumber sampling used only to compute the artificial profile, $\Delta k_{y}$ the wavenumber step and $\theta_{m}$ are independent random phase angles uniformly distributed in the interval $[0,2 \pi]$. The artificial track profile is generated from PSD function according to ISO 8608 [31]:

$$
\tilde{S}_{u_{w / r}}\left(k_{y}\right)=\tilde{S}_{u_{w / r}}\left(k_{y 0}\right)\left(\frac{k_{y}}{k_{y 0}}\right)^{-w}
$$

\subsection{Multi-body-Finite element-Boundary element coupling}

The boundary element equation 1 can be split in two parts, one for the nodes on the interface with the finite element sub-region and another for the nodes not shared with any other sub-region. Sub-index $c$ indicates the degrees of freedom associated with the interface and the sub-index $b$ the other boundary element degrees of freedom. The forces of all boundary element nodes are computed from their corresponding nodal tractions. Thus equation 1 can be rewritten as follows:

$$
\begin{aligned}
& {\left[\begin{array}{cc}
\mathbf{H}_{c c}^{n n} & \mathbf{H}_{c b}^{n n} \\
\mathbf{H}_{b c}^{n n} & \mathbf{H}_{b b}^{n n}
\end{array}\right]\left[\begin{array}{c}
\mathbf{u}_{c}^{n} \\
\mathbf{u}_{b}^{n}
\end{array}\right]=\left[\begin{array}{ll}
\widehat{\mathbf{G}}_{c c}^{n n} & \widehat{\mathbf{G}}_{c b}^{n n} \\
\widehat{\mathbf{G}}_{b c}^{n n} & \widehat{\mathbf{G}}_{b b}^{n n}
\end{array}\right]\left[\begin{array}{c}
\mathbf{f}_{c}^{n} \\
\mathbf{f}_{b}^{n}
\end{array}\right]+} \\
& \sum_{m=1}^{n-1}\left(\left[\begin{array}{cc}
\widehat{\mathbf{G}}_{c c}^{n m} & \widehat{\mathbf{G}}_{c b}^{n m} \\
\widehat{\mathbf{G}}_{b c}^{n m} & \widehat{\mathbf{G}}_{b b}^{n m}
\end{array}\right]\left[\begin{array}{c}
\mathbf{f}_{c}^{m} \\
\mathbf{f}_{b}^{m}
\end{array}\right]-\left[\begin{array}{ll}
\mathbf{H}_{c c}^{n m} & \mathbf{H}_{c b}^{n m} \\
\mathbf{H}_{b c}^{n m} & \mathbf{H}_{b b}^{n m}
\end{array}\right]\left[\begin{array}{l}
\mathbf{u}_{c}^{m} \\
\mathbf{u}_{b}^{m}
\end{array}\right]\right) \exp [-2 \pi \alpha(n-m) \Delta t]
\end{aligned}
$$

where:

$$
\mathbf{f}=\left[\sum_{a=1}^{A} \int_{\Gamma} \mathbf{N}^{T} \mathbf{N} d \Gamma\right] \mathbf{p}=\mathbf{L} \mathbf{p} \quad \text { and } \quad \hat{\mathbf{G}}=\mathbf{G L}^{-1}
$$

with $\mathbf{N}$ being the shape functions vector. Summation extends over the elements $A$ that contain the boundary element node.

The finite element equation 2 can also be split as follows,

$$
\left[\begin{array}{ll}
\mathbf{D}_{c c}^{n n} & \mathbf{D}_{c f}^{n n} \\
\mathbf{D}_{f c}^{n n} & \mathbf{D}_{f f}^{n n}
\end{array}\right]\left[\begin{array}{l}
\mathbf{u}_{c}^{n} \\
\mathbf{u}_{f}^{n}
\end{array}\right]=\left[\begin{array}{l}
\mathbf{f}_{c}^{n} \\
\mathbf{f}_{f}^{n}
\end{array}\right]+\left[\begin{array}{c}
\mathbf{f}_{c}^{n-1} \\
\mathbf{f}_{f}^{n-1}
\end{array}\right]
$$

where sub-index $c$ indicates the degrees of freedom are shared with the boundary element sub-region and $f$ stands for the rest of the finite element degrees of freedom.

Coupling boundary element and finite element sub-regions entails satisfying equilibrium and compatibility conditions at the interface between both regions. To do so, there are different procedures [32, 33]. Usually, domain formulations in time coupling are performed directly by writing boundary element equations and finite element equations in a single global system, together with the equilibrium and compatibility 
equations $[24,25,26]$. Thus,

$$
\begin{gathered}
{\left[\begin{array}{cccc}
\mathbf{H}_{c c}^{n n} & \mathbf{H}_{c b}^{n n} & -\widehat{\mathbf{G}}_{c c}^{n n} & \mathbf{0} \\
\mathbf{H}_{b c}^{n n} & \mathbf{H}_{b b}^{n n} & -\widehat{\mathbf{G}}_{b c}^{n n} & \mathbf{0} \\
\mathbf{D}_{c c} & \mathbf{0} & -\mathbf{I} & \mathbf{D}_{c f} \\
\mathbf{D}_{f c} & \mathbf{0} & \mathbf{0} & \mathbf{D}_{f f}
\end{array}\right]\left[\begin{array}{c}
\mathbf{u}_{c}^{n} \\
\mathbf{u}_{b}^{n} \\
\mathbf{f}_{c}^{n} \\
\mathbf{u}_{f}^{n}
\end{array}\right]=\left[\begin{array}{c}
\widehat{\mathbf{G}}_{c b}^{n n} \mathbf{f}_{b}^{n} \\
\widehat{\mathbf{G}}_{b b}^{n n} \mathbf{f}_{b}^{n} \\
\mathbf{0} \\
\mathbf{f}_{f}^{n}
\end{array}\right]+} \\
{\left[\begin{array}{c}
\sum_{m=1}^{n-1}\left(\widehat{\mathbf{G}}_{c c}^{n m} \mathbf{f}_{c}^{m}+\widehat{\mathbf{G}}_{c b}^{n m} \mathbf{f}_{b}^{m}-\mathbf{H}_{c c}^{n m} \mathbf{u}_{c}^{m}-\mathbf{H}_{c b}^{n m} \mathbf{u}_{b}^{m}\right) \exp [-2 \pi \alpha(n-m) \Delta t] \\
\sum_{m=1}^{n-1}\left(\widehat{\mathbf{G}}_{b c}^{n m} \mathbf{f}_{c}^{m}+\widehat{\mathbf{G}}_{b b}^{n m} \mathbf{f}_{b}^{m}-\mathbf{H}_{b c}^{n m} \mathbf{u}_{c}^{m}-\mathbf{H}_{b b}^{n m} \mathbf{u}_{b}^{m}\right) \exp [-2 \pi \alpha(n-m) \Delta t] \\
\mathbf{f}_{c}^{n-1} \\
\mathbf{f}_{f}^{n-1}
\end{array}\right.}
\end{gathered}
$$

This system of equations is used in the present model to represent the track and the structures (finite elements) and the soil (boundary elements).

Equation 3 is introduced into the boundary element-finite element system of equations 10 taking into account the compatibility displacements and equilibrium forces at the wheel-rail contact points. Thus, the vehicle-track interaction forces, considering the train-track-soil dynamic interaction, can be taken into account in the problem and evaluated if required.

As the vehicle moves along the track according to its speed, the contact point between the vehicle and the rail changes as time goes on (figure 4). A moving node is created in the rail to couple the vehicle and the track. So the track mesh including the rail changes at each time step and the obtained finite element system of equations becomes non-linear because mass, damping and stiffness matrices vary at each time step. Nevertheless, the time domain formulation allows one to solve the non-linear system of equations using, for example, the methodology presented in reference $\underline{[34]}$.

[Figure 4 about here.]

\section{Quasi-static and dynamic excitation mechanisms}

In this section, the different components of the excitation mechanism are studied. Quasi-static and dynamic effects due to the vehicle behaviour, sleeper discrete support and the rail and wheel unevenness are all analyzed.

[Figure 5 about here.]

Figure 5a shows the cross section of a classical ballast track that it is represented by the mesh shown in figure 5b. The ballast track is represented by 1740 finite elements: the rails and the sleepers are modelled as 872 Euler-Bernoulli beam elements, the rail-pads are modelled as 292 spring-damper elements, and the ballast is modelled using 576 solid elements. The finite element model has 8253 degrees of freedom. The track 
is composed of two UIC60 rails with a bending stiffness $E I=6.45 \times 10^{6} \mathrm{Nm}^{2}$ and a mass per unit length $m=60.3 \mathrm{~kg} / \mathrm{m}$ for each rail. The rail-pads have a $10 \mathrm{~mm}$ thickness and their stiffness and damping values are $k_{r p}=150 \times 10^{6} \mathrm{~N} / \mathrm{m}$ and $c_{r p}=13.5 \times 10^{3} \mathrm{Ns} / \mathrm{m}$, respectively. The prestressed concrete monoblock sleepers have a length $l=2.50 \mathrm{~m}$, a width $w=0.235 \mathrm{~m}$, a height $h=0.205 \mathrm{~m}$ (under the rail) and a mass $m=300 \mathrm{~kg}$. A distance $d=0.6 \mathrm{~m}$ between the sleepers is considered. The ballast has a density $\rho=1500 \mathrm{~kg} / \mathrm{m}^{3}$, a Poisson ratio $\nu=0.2$, and a Young's modulus equal to $E=200 \times 10^{6} \mathrm{~N} / \mathrm{m}^{2}$. The width of the ballast equals $2.92 \mathrm{~m}$ and the height $h=1.00 \mathrm{~m}$.

The track is assumed to be located at the surface of a homogeneous half-space that represents the soil, with a S-wave velocity $C_{s}=150.0 \mathrm{~m} / \mathrm{s}$, a P-wave velocity $C_{p}=299.8 \mathrm{~m} / \mathrm{s}$, and a Rayleigh wave velocity $C_{R}=139.7 \mathrm{~m} / \mathrm{s}$. The soil discretization is shown in figures $5 \mathrm{~b}$ and $5 \mathrm{c}$. The soil surface is discretized into 864 quadrilateral nine-node quadratic elements. The mesh has a total length of $86 \mathrm{~m}$ and a width of $29 \mathrm{~m}$. The boundary element model has 11472 degrees of freedom. It is worth mentioning that to validate the mesh in figure $5 \mathrm{~b}$, the problem of a point load travelling at constant speed on the surface of half-space without track was studied first using the same mesh. A good agreement between numerical and analytical solution was obtained for the same velocities and properties of the present study. Results of that experiment can be seen in [22]. The size of the elements used in the discretization of Figure 5b is small enough to represent the soil surface motion for an axle load at the speeds of interest. It should be taken into account that a $15 \times 15$ element subdivision is used to carry out numerical integration over the boundary elements. The time step depends on the train speed and it allows the representation of the quasi-static load and the rail and wheel irregularities. Previous boundary element analysis of soil vibration problems show that a mesh several times as wide as the loading zone is enough to obtain accurate results in an area around the loading zone [21]. In the present study, a mesh width of $29 \mathrm{~m}$ was chosen in order to be able to measure soil surface displacements up to $11.8 \mathrm{~m}$ from the track axis. A good representation of the soil surface displacements at larger distances would require a wider mesh. The running time of the model is about 44 hours on a personal computer (PC) with $3.48 \mathrm{~GB}$ RAM and a $3.06 \mathrm{GHz}$ processor.

\subsection{Quasi-static and parametric excitation mechanisms}

The quasi-static response due to the passage of a single axle train can be obtained from the equations 3 and 10 with the unevenness term $u_{w / r}$ equal to zero. Since the sleepers are included in the model, the dynamic effects due to discrete rail support (figure 4) are considered implicitly.

Figure 6 shows the time history of the vertical displacement and the vertical velocity and the frequency content of the vertical velocity at the rail for a single axle travelling at $v=298 \mathrm{~km} / \mathrm{h}$ computed from a model where only a moving force is considered and from the multi-body model shown in figure 3 . The characteristics of the axle are the following: mass of the car body $\bar{M}_{c}=12000 \mathrm{~kg}$, bogie mass $\bar{M}_{b}=1520 \mathrm{~kg}$, and the unsprung mass of the wheelset $\bar{M}_{w}=2003 \mathrm{~kg}$; the primary suspension, $\bar{k}_{1}=1.4 \times 10^{6} \mathrm{~N} / \mathrm{m}$ and 
$\bar{c}_{1}=10 \times 10^{3} \mathrm{~N} / \mathrm{m} / \mathrm{s}$, and the secondary suspension, $\bar{k}_{2}=0.41 \times 10^{6} \mathrm{~N} / \mathrm{m}$ and $\bar{c}_{2}=24 \times 10^{3} \mathrm{~N} / \mathrm{m} / \mathrm{s}$. The total axle force is estimated as $152 \mathrm{kN}$.

[Figure 6 about here.]

The computed displacement shown in figure $6 \mathrm{a}$ is completely symmetric and, therefore, it is worth to point out that the use of a full space fundamental solution with a bounded free surface discretization does not produce significant spurious noise caused by diffraction at the limits of the discretized area. The time histories of the vertical displacement and velocity at the rail computed from both models have similar values. However, the computed frequency content of the vertical velocity present differences due the inertia is being neglected in the moving force model. Both models lead to the same solution only when the axle speed is low or if the primary vertical stiffness tends to zero [35]. Therefore, the vehicle model should be considered to predict the quasi-static response.

[Figure 7 about here.]

Figure 7 shows the time history and the frequency content of the vertical displacement at the vehicle due to the moving axle and the parametric excitation due to the discrete supports of the rails for a single axle travelling at $v=298 \mathrm{~km} / \mathrm{h}$ and at $v=36 \mathrm{~km} / \mathrm{h}$. The sleepers positions are also shown. In figure 7a it is clearly observed that the maximum displacements at the body car occur when the axle is approximately in the middle of the sleeper distance and the minimum when the axle is approximately on the sleeper in accordance to the lower and higher stiffness of the track in those zones, respectively. Figures $7 \mathrm{c}, \mathrm{d}$ show a peak corresponding to the vehicle resonance frequency at about $13 \mathrm{~Hz}$. Figure $7 \mathrm{c}$ shows sharp peaks associated with the sleeper passing frequency $f_{s}=v / d=16.7 \mathrm{~Hz}$ and its higher order harmonics. As the train speed increase (figures $7 \mathrm{~b}, \mathrm{~d}$ ), the parametric excitation due to the sleeper discrete support effect goes to higher frequencies and its effect at the studied frequency range can be neglected. Figure $7 \mathrm{~d}$ shows a peak at $f_{s}=v / d=137.9 \mathrm{~Hz}$.

\subsection{Dynamic excitation mechanism}

In order to investigate the behaviour of the coupled vehicle-track-soil system due to rail and wheel unevenness, the dynamic response due to the passage of a single axle train can be obtained from equations 3 and 10 if the acceleration due to gravity is neglected. Again, the sleepers are included in the model and, therefore the dynamic effects due to discrete rail support are also taken into account. An artificial track profile is generated from the PSD function according to ISO 8608 by equation 6 with $k_{y 0}=1 \mathrm{rad} / \mathrm{m}, w=3.5$ as commonly assumed for railway unevenness, and a value of $\tilde{S}_{u_{w / r}}\left(k_{y 0}\right)=2 \pi \times 10^{-8} \mathrm{~m}^{3}$.

[Figure 8 about here.] 
It is commonly adopted in the literature $[8, \underline{10}, \underline{36]}$ that the influence of the suspended mass can be neglected, and the vehicle's unsprung mass is the only component that affects the dynamic loads due to the rail and wheel unevenness. The consistency of the previous hypothesis is studied from the results presented in figure 8 where the time history and the frequency content of the vertical displacement at the rail due to unevenness (figure 9a) for a single axle travelling at $v=298 \mathrm{~km} / \mathrm{h}$ computed from the unsprung mass model and the multi-body model, respectively, are shown. Both models lead to a similar response for frequencies higher than $10 \mathrm{~Hz}$ approximately. Therefore, the model could be simplified as at frequencies of more than a few Hertz [36] since the vehicle's primary and secondary suspension isolate the body and the bogie from the wheelset. A maximum occurs at the rail displacement between 10 and $40 \mathrm{~Hz}$ for both models that corresponds to the resonance frequency of the vehicle model (grey line) or the unsprung mass model (black line) on the track. However, at lower frequencies, the suspended mass should be taken into account in order to predict the track and soil response accurately.

[Figure 9 about here.]

Figure $9 \mathrm{~b}$ shows the frequency content of the vertical velocity at the vehicle due to the unevenness shown in figure $9 \mathrm{a}$ for a single axle travelling at $v=298 \mathrm{~km} / \mathrm{h}$ computed from the multi-body model. The wheel response shows the contributions due to the resonance frequency of the vehicle model on the track and the dynamic behaviour of the vehicle. The primary suspension filters the bogie response which appear at the

bogie resonance frequency, $\sqrt{\bar{k}_{1} /\left(\bar{M}_{c}+\bar{M}_{b}\right)} / 2 / \pi=1.62 \mathrm{~Hz}$. The secondary suspension isolates the body car and the frequency content of its response is approximately at $\sqrt{\bar{k}_{2} /\left(\bar{M}_{c}\right)} / 2 / \pi=0.93 \mathrm{~Hz}$.

\section{Experimental validation}

In this section, the numerical model is experimentally validated by comparison of the computed results with existing experimental data recorded at two HST lines: Córdoba-Málaga [23] and Brussels-Paris [37]. Both, experimental and numerical results were filtered using a high-pass digital filter with a cut-off frequency $f_{p}=3 \mathrm{~Hz}\left(f_{s}=2.5 \mathrm{~Hz}\right)$.

\subsection{First case: Sub-Rayleigh regime}

First, the numerical model is validated by comparison of the computed results with those measured on the HST line between Córdoba and Málaga presented by Galvín and Domínguez [23]. The track between Córdoba and Málaga is composed of two UIC60 rails with a bending stiffness $E I=6.45 \times 10^{6} \mathrm{Nm}^{2}$ and a mass per unit length $m=60.3 \mathrm{~kg} / \mathrm{m}$ for each rail. The rail-pads have a thickness of $10 \mathrm{~mm}$ and their stiffness and damping values are $k_{r p}=150 \times 10^{6} \mathrm{~N} / \mathrm{m}$ and $c_{r p}=13.5 \times 10^{3} \mathrm{Ns} / \mathrm{m}$, respectively [38]. The prestressed concrete monoblock sleepers have a length $l=2.60 \mathrm{~m}$, a width $w=0.235 \mathrm{~m}$, a height $h=0.205 \mathrm{~m}$ (under 
the rail) and a mass $m=300 \mathrm{~kg}$. A distance $d=0.6 \mathrm{~m}$ between the sleepers is considered. The ballast has a density $\rho=1500 \mathrm{~kg} / \mathrm{m}^{3}$, a Poisson ratio $\nu=0.2$, and a Young's modulus equal to $E=450 \times 10^{6} \mathrm{~N} / \mathrm{m}^{2}$. The width of the ballast equals $2.92 \mathrm{~m}$ and the height $h=1.00 \mathrm{~m}$.

The dynamic properties of the soil on which the measurements were taken were obtained experimentally by the SASW (Spectral Analysis of Surface Waves) testing procedure since only non-intrusive testing methods and instruments could be used. The results present in reference [23] show that the soil can be represented by an equivalent homogenous half-space with an S-wave velocity $C_{s}=150.0 \mathrm{~m} / \mathrm{s}$, a P-wave velocity $C_{p}=299.8 \mathrm{~m} / \mathrm{s}$, and a Rayleigh wave velocity $C_{R}=139.7 \mathrm{~m} / \mathrm{s}$. The Barkan formula for homogeneous soil [28] was used to estimate the soil attenuation coefficient $\alpha$ from experimental records, which equals 1.1.

The carriage length $L_{t}$, the distance $L_{b}$ between bogies, the axle distance $L_{a}$, the total axle mass $M_{t}$ and the unsprung axle mass $M_{u}$ of all the carriages of the AVE HST are shown in figure 2 and Table 1. Table 2 shows the primary vertical stiffness, $k_{1}$, the primary vertical viscous damping, $c_{1}$, the secondary vertical stiffness, $k_{2}$, and the secondary vertical viscous damping, $c_{2}$, of the HST.

[Table 1 about here.]

[Table 2 about here.]

The considered train speed equals $v=298 \mathrm{~km} / \mathrm{h}$ and the Rayleigh wave velocity in the soil is $C_{R}=139.7 \mathrm{~m} / \mathrm{s}$. In this case, the train speed is lower than the Rayleigh wave velocity in the soil. Therefore, according to the results present in previous studies $\underline{[7,10]}$ the contribution of the dynamic loading mechanisms to the vertical motion of the free field points should be more important than the quasi-static loading mechanism. The track unevenness was not measured in the site where the induced vibrations were recorded. Then the ISO 8608 is used to generate artificial track profiles from the PSD function by equation 6. As different samples of track unevenness yield different predictions of the track and free field response [10], six samples of unevenness are generated by means of equation 5 as a superposition of harmonic functions with the same amplitude, but a different random phase, to make the experimental validation of the numerical model. Figure 9a shows the one-third octave band spectra of the six samples of unevenness. The six samples generate the same one-third octave band spectra [10].

[Figure 10 about here.]

[Figure 11 about here.]

[Figure 12 about here.]

Figures 10, 11 and 12 show the comparison between the time histories, frequency contents and one-third octave band spectra, respectively, of the vertical velocity at the sleeper and three points in the free field. 
Figure 12 shows the computed results for the six samples of unevenness. The computed results agree quite well with the experimental results at the sleeper, where the quasi-static contribution is dominant and the response does not depend on the unevenness. In the free field, where the contribution of the dynamic loading mechanisms to the vertical motion is important, the comparison between the computed and the experimental values show a reasonable agreement, but it is not as good as for the sleeper point. Moreover, the correlation depends on the track profile as shown in figure 12. This fact can be explained by taking into account that it is not possible to represent the actual unevenness from equation 5 which represent the geometry only in a random sense. The methodology presented by Lombaert and Degrande [10] leads an ensemble average that can be used to avoid the random sense of the unevenness. In any case, it can be concluded from the present comparison, and that presented in reference [23], that the numerical model has been significantly improved by including the dynamic excitation mechanism. The discrepancies between computed and measured velocities can be also explained accounting for the soil representation. In the present model, the soil is represented by a homogeneous halfspace. However, the actual soil presents strata that are not considered in the model.

\subsection{Second case: Super-Rayleigh regime}

Secondly, the proposed numerical model is validated using the values recorded by Degrande and Schillemans on the HST line between Brussels and Paris [37] considering a train passage where the train speed is higher than the Rayleigh wave velocity and the S-wave velocity in the soil. The track has the same properties that the previously studied track except in this case the sleepers have a length $l=2.50 \mathrm{~m}$ and the ballast has a Young's modulus equal to $E=250 \times 10^{6} \mathrm{~N} / \mathrm{m}^{2}$. The results present in reference [6] show that the soil can be represented by an equivalent homogenous half-space with an S-wave velocity $C_{s}=80.0 \mathrm{~m} / \mathrm{s}$, a P-wave velocity $C_{p}=150.0 \mathrm{~m} / \mathrm{s}$, and a Rayleigh wave velocity $C_{R}=74.1 \mathrm{~m} / \mathrm{s}$. The Barkan formula for homogeneous soil [28] was used to estimate the soil attenuation coefficient $\alpha$, and this equals 4.4. The configuration, geometrical and mass characteristics of the Thalys HST can be seen in reference [37] and they are similar to those previously presented.

The considered train speed equals $v=315 \mathrm{~km} / \mathrm{h}$ and the Rayleigh wave velocity in the soil is $C_{R}=$ $74.1 \mathrm{~m} / \mathrm{s}$. In this case, the train speed is higher than the Rayleigh wave velocity in the soil. Therefore, it prevails the contribution of the quasi-static loading mechanisms to the vertical motion of the free field points [7]. An artificial track profile is generated using ISO 8608 to take into account the dynamic load.

Figures 13, 14 and 15 show the comparison between the time histories, frequency contents and one-third octave band spectra of the vertical velocity at the sleeper and three observations points in the free field for a train speed equals $v=315 \mathrm{~km} / \mathrm{h}$. Both, quasi-static and dynamic excitation, mechanisms are considered. However, the quasi-static excitation dominates the free field response as is clearly shown in figure 15 and the dynamic contribution could be neglected. The computed results show a good agreement with those 
experimentally recorded. A good agreement was also obtained in reference [6]. The frequency content and the one-third octave band spectra of the free field response (Figures 14 and 15) show peaks in the bogie passing frequency $f_{b}=4.7 \mathrm{~Hz}$ and in the axle passing frequency $f_{a}=29.2 \mathrm{~Hz}$.

[Figure 13 about here.]

[Figure 14 about here.]

[Figure 15 about here.]

\section{Vibrations induced by high-speed train passage in a transition zone between ballast and slab track}

The use of ballastless slab track is significantly increasing in HST line track technology. This development is due to the fact that slab track has some structural and operational advantages over ballasted track $[30,39]$. In addition, the floating slab tracks can be used to control ground-borne vibrations generated by railway traffic $[40, \underline{41}, \underline{42,43]}$. The growing interest in this relatively new track technology in recent years, calls for additional studies to be conducted. Because of that, the proposed numerical model is used in this section to study the dynamic behaviour of a transition zone between a ballast track (figure 16a) and a slab track (figure 16b) which is frequently located at tunnel entrances or near railway stations.

[Figure 16 about here.]

Figure 16a shows the ballasted track system considered. The track is composed of two layers: a ballast layer over a sub-ballast layer. The ballast has a density $\rho=1500 \mathrm{~kg} / \mathrm{m}^{3}$, a Poisson ratio $\nu=0.2$, and a Young's modulus $E=280 \times 10^{6} \mathrm{~N} / \mathrm{m}^{2}$. The sub-ballast layer has a density $\rho=1500 \mathrm{~kg} / \mathrm{m}^{3}$, a Poisson ratio $\nu=0.2$, and a Young's modulus $E=140 \times 10^{6} \mathrm{~N} / \mathrm{m}^{2}$. The rail-pads have a thickness of $10 \mathrm{~mm}$ and the stiffness and damping values are $k_{r p}=150 \times 10^{6} \mathrm{~N} / \mathrm{m}$ and $c_{r p}=13.5 \times 10^{3} \mathrm{Ns} / \mathrm{m}$.

The considered slab track system is shown in figure 16b. The track is composed of two UIC60 rails supported by prestressed concrete monoblock sleepers separated by $d=0.60 \mathrm{~m}$. The concrete slab has a density $\rho=2500 \mathrm{~kg} / \mathrm{m}^{3}$, a Poisson ratio $\nu=0.2$, and a Young's modulus $E=34 \times 10^{9} \mathrm{~N} / \mathrm{m}^{2}$. A hydraulic subbase is placed below the concrete slab in order to reduce gradually the transmitted forces from the track to the subgrade. The hydraulic subbase has a density $\rho=2500 \mathrm{~kg} / \mathrm{m}^{3}$, a Poisson ratio $\nu=0.2$, and a Young's modulus $E=10 \times 10^{9} \mathrm{~N} / \mathrm{m}^{2}$, and the same width as the concrete slab. Three different rail pads have been considered with the properties shown in table 3 .

[Table 3 about here.]

[Figure 17 about here.] 
Slab tracks have a higher stiffness as compared to ballasted tracks and, because of that a transition zone between ballasted and slab track must be carefully designed to make a gradual change of the stiffness to ensure track quality and passenger comfort. Figure 17a shows the geometry of the transition zone between ballasted and slab track. In the transition slab track zone the thickness of the hydraulic subbase layer increases from $0.3 \mathrm{~m}$ to $0.46 \mathrm{~m}$. After that, the hydraulic subbase layer replaces the sub-ballast layer under the ballast layer in the transition ballasted track zone. By doing so, the stiffness changes between the different parts of the transition zone are smoother. The discretization of the transition zone employed is shown in figure $17 \mathrm{~b}$. The track and soil response are studied at four sections. Section A is located in the ballasted track $3 \mathrm{~m}$ before the transition zone. Section B corresponds to the middle of the ballasted transition zone, and section $\mathrm{C}$ to the middle of slab track transition. Finally, section $\mathrm{D}$ is located in the slab track $9 \mathrm{~m}$ from the transition zone.

The track is assumed to be located at the surface of a homogeneous half-space that represents the soil. Three different soils have been considered with the properties shown in table 4 .

[Table 4 about here.]

Figure 18 shows the running RMS value and one-third octave band spectra of the vertical velocity at the rail, at the sleeper and at a point in the free field located at $11.8 \mathrm{~m}$ from the track axis for a train speed $v=298 \mathrm{~km} / \mathrm{h}$ travelling on the slab track for the three different rail pads. The track is located on the medium soil. Rail and sleeper have a similar behaviour where the quasi-static contribution is predominated. At low frequencies the velocity is higher as the rail pad stiffness decreases. The bogie passing frequency $f_{b}=4.4 \mathrm{~Hz}$ and the axle passing frequency $f_{a}=27.6 \mathrm{~Hz}$ lead the medium frequency range. In the free field a different behaviour is obtained. The soil response is dominated by the contribution of the dynamic loading mechanisms at frequencies higher than $31.5 \mathrm{~Hz}$ and presents higher values as the rail pad stiffness increases. The rail pads play an important role in vibration induced due to train passage on non-ballasted tracks and softer rail pads could be used in order to mitigate the free field response.

[Figure 18 about here.]

Figures 19 and 20 show the running RMS value and one-third octave band spectra of the vertical velocity at the rail, at the sleeper and at a point in the free field located at $11.8 \mathrm{~m}$ from track axis for a train speed $v=298 \mathrm{~km} / \mathrm{h}$ travelling on the transition zone for the three different soils. Medium rail pads are considered. For the soft soil the train is running faster than Rayleigh wave velocity (super-Rayleigh regime). So, a clear amplification is observed for the ballasted track in section A. Then, the quasi-static contribution prevails in the track and free field responses as the in HST Brussels-Paris case, previously studied. However, for the slab track in section D only a light increase is observed when the softer soil is considered. The super-critical behaviour which takes place in the ballasted track zone does not appear in the slab track due to higher 
stiffness of the system. For the slab track, the low frequency response depends on the soil properties and higher levels are obtained for softer soils. In the free field response the quasi-static excitation at medium frequencies gains importance when the S-wave velocity in the soil goes to lower values.

[Figure 19 about here.]

[Figure 20 about here.]

Finally, an analysis of the transition zone between ballast and slab track is made using models which consider an invariant geometry with respect to the track direction, and the obtained results are compared with those previously presented. Four independent track-soil models are used to compute the response in each section. Figure 21 compares the results from the transition zone model (using the present three dimensional model) and a two-and-a-half model (using the present three dimensional model with track and soil invariant in the track direction). The results obtained for both models in section A are similar. As the train goes into the transition zone the vertical stiffness of the track is modified and a transient response appears due to the inertial forces induced by the vehicle mass. The correlation between the computed results from both models in the transition zone present is not good and three dimensional models should be used to obtain an accurate response for these problems. The transient response produced by the stiffness track change disappears as the train travels along the slab track, and far from the transition zone both models tend to the same results.

[Figure 21 about here.]

\section{Conclusions}

This paper is intended to develop a general numerical model for the analysis of vibrations due to HST and their effects on nearby structures. The numerical model is based on the three dimensional finite element and boundary element formulations in the time domain. As compared to two-and-a-half domain solutions, the present formulation can take into account local soil discontinuities, underground constructions such as underpasses, and coupling with nearby structures that break the uniformity of the geometry along the track line. Track and other structures are modelled using the finite element method and their non-linear behaviour can be considered because a time domain formulation is employed. The soil is represented using the boundary element method, where a full-space fundamental solution is used in combination with quadratic boundary elements. The train vehicle is modelled as a multi-body and, therefore, the quasi-static and the dynamic excitation mechanisms can be considered, taking into account the dynamic effects due to sleeper discrete support and the wheel and rail irregularities.

The influence of the vehicle model in the quasi-static and dynamic responses has been studied. The vehicle inertia should be considered to predict accurately the quasi-static response. For the dynamic response, the suspended mass should be taken into account in order to predict the track and soil response at low frequencies. 
The numerical model has been experimentally validated by comparison with existing experimental records taken in two HST lines: the Córdoba-Málaga HST line $\underline{[23]}$ and the Brussels-Paris HST line $\underline{[37]}$. In the first case, the train speed was lower than the Rayleigh wave velocity in the soil. Thus, quasi-static and dynamic contributions are important to reproduce the actual problem. Different samples of track unevenness yield different predictions of the track and free field response due to the dynamic excitation [10]. Then, six samples of unevenness are generated to make the experimental validation of the numerical model. In the second case, the train speed is higher than the Rayleigh wave velocity in the soil and, therefore, the quasi-static contribution dominates the track and free field response. In both cases, the correlation between experimental and computed results is quite good.

The dynamic behaviour of a transition zone between a ballast track and a slab track has also been studied by the present three dimensional model. The computed results have been compared with those obtained from models which consider an invariant geometry with respect to the track direction. It has been concluded, in this case, that a three dimensional model including the non-linearity of the track should be used to obtain accurate responses.

\section{Acknowledgements}

This research is financed by the Ministerio de Educación y Ciencia of Spain under the research project BIA2007-67612-C02-02 and by the CEDEX under the research project PT-2006-024-19CCPM. The financial support is gratefully acknowledged.

\section{References}

[1] I. Barrón, High Speed lines in the World, UIC High Speed Department, 2008.

[2] K. Adolfsson, B. Andréasson, P.-E. Bengtson, A. Bodare, C. Madshus, R. Massarch, G. Wallmark, P. Zackrisson, High speed lines on soft ground. Evaluation and analyses of measurements from the West Coast Line, Technical Report, Banverket, 1999.

[3] M. Heckl, G. Hauck, R. Wettschureck, Structure-borne sound and vibration from rail traffic, Journal of Sound and Vibration 193 (1996) 175-184.

[4] V. Krylov, Generation of ground vibrations by superfast trains, Applied Acoustics 44 (1995) 149-164.

[5] G. Degrande, Free field vibrations during the passage of a high speed train: experimental results and numerical predictions, in: V. Krylov (Ed.), Noise and vibration from high-speed trains, Thomas Telford Publishing, London, 2001.

[6] P. Galvín, J. Domínguez, High-speed train-induced ground motion and interaction with structures, Journal of Sound and Vibration 307 (2007) 755-777.

[7] X. Sheng, C.J.C. Jones, D.J. Thompson, A comparison of a theoretical model for quasi-statically and dynamically induced environmental vibration from trains with measurements, Journal of Sound and Vibration 267 (2003) 621-635.

[8] G. Lombaert, G. Degrande, J. Kogut, S. François, The experimental validation of a numerical model for the prediction of railway induced vibrations, Journal of Sound and Vibration 297 (2006) 512-535.

[9] L. Auersch, The effect of critically moving loads on the vibrations of soft soils and isolated railway tracks, Journal of Sound and Vibration 310 (2008) 587-607. 
[10] G. Lombaert, G. Degrande, Ground-borne vibration due to static and dynamic axle loads of InterCity and high-speed trains, Journal of Sound and Vibration 319 (2009) 1036-1066.

[11] A.V. Metrikine, S.N. Verichev, J. Blauwendraad, Stability of a two-mass oscillator moving on a beam supported by a visco-elastic half-space, International Journal of Solids and Structures (2005) 1187-1207.

[12] A.V. Metrikine, K. Popp, Instability of vibrations of an oscillator moving along a beam on an elastic half-space, European Journal of Mechanics, A/Solids 18 (1999) 331-349.

[13] H.A. Dieterman, A.V. Metrikine, The equivalent stiffness of a halfspace interacting with a beam. Critical velocities of a moving load along the beam, European Journal of Mechanics, A/Solids 15 (1996) 67-90.

[14] L. Auersch, The excitation of ground vibration by rail traffic: theory of vehicle-track-soil interaction and measurements of high-speed lines, Journal of Sound and Vibration 284 (2005) 103-132.

[15] H. Takemiya, X. Bian, Substructure simulation of inhomogeneous track and layered ground dynamic interaction under train passage, Journal of Engineering Mechanics 131 (2005) 699-711.

[16] A.V. Metrikine, K. Popp, Vibration of a periodically supported beam on an elastic half-space, European Journal of Mechanics, A/Solids 18 (1999) 679-701.

[17] X. Sheng, C.J.C. Jones, D.J. Thompson, A theoretical model for ground vibration from trains generated by vertical track irregularities, Journal of Sound and Vibration 272 (2004) 937-965.

[18] X. Sheng, C.J.C. Jones, D. J. Thompson, Prediction of ground vibration from trains using the wavenumber finite and boundary element methods, Journal of Sound and Vibration 293 (2006) 575-586.

[19] H. Xia, Y.M. Cao, G. De Roeck, Theoretical modeling and characteristic analysis of moving-train induced ground vibrations, Journal of Sound and Vibration 329 (2010) 819-832.

[20] O.C. Zienkiewicz, The Finite Element Method, McGraw-Hill Company, London, 1977.

[21] J. Domínguez, Boundary elements in dynamics, Computational Mechanics Publications and Elsevier Applied Science, Southampton, 1993.

[22] P. Galvín, J. Domínguez, Analysis of ground motion due to moving surface loads induced by high-speed trains, Engineering analysis with boundary elements 30 (2007) 931-941.

[23] P. Galvín, J. Domínguez, Experimental and Numerical Analysis of Vibrations Induced by High-Speed Trains on the Córdoba-Málaga Line, Soil Dynamics and Earthquake Engineering 29 (2009) 641-657.

[24] D.L. Karabalis, D.E. Beskos, Dynamic response of 3D flexible foundations by time domain BEM and FEM, Soil Dynamics and Earthquake Engineering 4 (1985) 91-101.

[25] O. von Estorff, M.J. Prabucki, Dynamic response in the time domain by coupled boundary and finite elements, Computational Mechanics 6 (1990) 35-46.

[26] M. Firuziaan, O. von Estorff, Transient 3D soil/structure interaction analyses including nonlinear effects, in H. Grundmann, G.I. Schuëller (Eds.), Structural dynamics-EURODYN2002, Lisse: Swets and Zeitlinger, 2002, 1291-1296.

[27] M. Marrero, J. Domínguez, Numerical behavior of time domain BEM for three-dimensional transient elastodynamic problems, Engineering analysis with boundary elements 27 (2002) 39-48.

[28] D.D. Barkan, Dynamics of Bases and Foundations, McGraw-Hill, New York, 1962.

[29] N.M. Newmark, A method of computation for structural dynamics, ASCE Journal of the Engineering Mechanics Division $85(1959) 67-94$.

[30] C. Esveld, Modern Railway Track, MRT Productions, Zaltbommel, 2001.

[31] International Organization for Standardization. ISO 8608:1995: Mechanical vibration - Road surface profiles - Reporting of measured data, 1995.

[32] D.E. Beskos, Boundary element methods in dynamic analysis, Applied Mechanics Review 40 (1987) 1-23.

[33] D.E. Beskos, Boundary element methods in dynamic analysis. Part II (1986-1996), Applied Mechanics Review 50 (1997) 
149-197.

[34] S.Y. Chang, Nonlinear error propagation analysis for explicit pseudodynamics algorithm, Journal of engineering mechanics ASCE 123 (2003) 841-850.

[35] A.V. Pesterev, L.A. Bergman, C.A. Tan, T.-C. Tsao, B. Yang, On asymptotics of the solution of the moving oscillator problem, Journal of Sound and Vibration 260 (2003) 519-536.

[36] K. Knothe, S.L. Grassie, Modelling of railway track and vehicle/track interaction at high frequencies, Vehicle System Dynamics 22 (1993) 209-262.

[37] G. Degrande, L. Schillemans, Free field vibrations during the passage of a Thalys HST at variable speed, Journal of Sound and Vibration 247 (2001) 131-144.

[38] GIF. Proyecto de Construcción de la Línea de Alta Velocidad Córdoba-Málaga, 1999.

[39] M.J.M.M. Steenbergen, A. V. Metrikine, C. Esveld, Assesment of design parameters of a slab track railway system from a dynamic viewpoint, Journal of Sound and Vibration 306 (2007) 361-371.

[40] G. Lombaert, G. Degrande, B. Vanhauwere, B. Vandeborght, S. François, The control of ground-borne vibrations from railway traffic by means of continuous floating slabs, Journal of Sound and Vibration 297 (2006) 946-961.

[41] G. Degrande, D. Clouteau, R. Othman, M. Arnst, H. Chebli, R. Klein, P. Chatterjee, B. Janssens, A numerical model for ground-borne vibrations from underground railway traffic based on a periodic finite element-boundary element formulation, Journal of Sound and Vibration 293 (2006) 645-666.

[42] S. Gupta, M.F.M. Hussein, G. Degrande, H.E.M. Hunt, D. Clouteau, A comparison of two numerical models for the prediction of vibrations from underground railway traffic, Soil Dynamics and Earthquake Engineering 27 (2007) 608-624.

[43] P. Galvín, A. Romero, J. Domínguez, Vibrations induced by HST passage on ballast and non-ballast tracks, Soil Dynamics and Earthquake Engineering (2010) doi:10.1016/j.soildyn.2010.02.004. 


\section{List of Figures}

1 The dynamic track-soil and soil-structure interaction problem. . . . . . . . . . . . . . . 21

2 Configuration of the Córdoba-Málaga HST. . . . . . . . . . . . . . . . . . . . . . . . . . 22

3 The multi-body model for an axle. . . . . . . . . . . . . . . . . . . . . . . 23

4 Vehicle and track model at: (a) time step i and (b) time step i+1. . . . . . . . . . . . . . . . 24

5 (a) Ballast system track, (b) Track and soil discretization and (c) Detail of the discretization. 25

6 (a) Time history of the vertical displacement and (b) vertical velocity and (c) frequency content of the vertical velocity at the rail for a single axle travelling at $v=298 \mathrm{~km} / \mathrm{h}$ computed from the moving force model (black line) and the multi-body model (grey line). . . . . . . . .

$7 \quad(\mathrm{a}, \mathrm{b})$ Time histories and $(\mathrm{c}, \mathrm{d})$ frequency contents of the vertical displacement at the body car (light grey line), bogie (dark grey line) and wheel (black line) due to sleeper discrete support for a single axle travelling at $(\mathrm{a}, \mathrm{c}) v=36 \mathrm{~km} / \mathrm{h}$ and $(\mathrm{b}, \mathrm{d}) v=298 \mathrm{~km} / \mathrm{h}$. The sleepers positions are shown as vertical grey lines. . . . . . . . . . . . . . . . . . .

8 (a) Time history and (b) frequency content of the vertical displacement at the rail for a single axle travelling at $v=298 \mathrm{~km} / \mathrm{h}$ computed from the unsprung mass model (black line) and the multi-body model (grey line) . . . . . . . . . . . . . . . . . . . . .

9 (a) One-third octave band spectra of the track unevenness and (b) the frequency content of the vertical velocity at the body car (black line), bogie (light grey line) and wheel (dark grey line) due to unevenness for a single axle travelling at $v=298 \mathrm{~km} / \mathrm{h}$ computed from the multi-body model. . . . . . . . . . . . . . . . . . . . . .

10 The experimental (black line) and computed (grey line) time history of the vertical velocity at (a) the sleeper and the free field at a distance of: (b) $3 \mathrm{~m}$; (c) $8 \mathrm{~m}$; and (d) $11.8 \mathrm{~m}$ from the axis track during the passage of the HST at a speed $v=298 \mathrm{~km} / \mathrm{h}$. . . . . . . . . . . . .

11 The experimental (black line) and computed (grey line) frequency content of the vertical velocity at (a) the sleeper and the free field at a distance of: (b) $3 \mathrm{~m}$; (c) $8 \mathrm{~m}$; and (d) $11.8 \mathrm{~m}$ from the axis track during the passage of the HST at a speed $v=298 \mathrm{~km} / \mathrm{h}$. . . . . . . . .

12 The experimental (black line) and computed for 6 samples of vertical track unevenness (grey lines) one-third octave band spectra of the vertical velocity at (a) the sleeper and the free field at a distance of: (b) $3 \mathrm{~m}$; (c) $8 \mathrm{~m}$; and (d) $11.8 \mathrm{~m}$ from the axis track during the passage of the HST at a speed $v=298 \mathrm{~km} / \mathrm{h} \ldots \ldots \ldots \ldots \ldots \ldots \ldots$

13 The experimental (black line) and computed (grey line) time history of the vertical velocity at (a) the sleeper and the free field at a distance of: (b) $4 \mathrm{~m}$; (c) $6 \mathrm{~m}$; and (d) $8 \mathrm{~m}$ from the

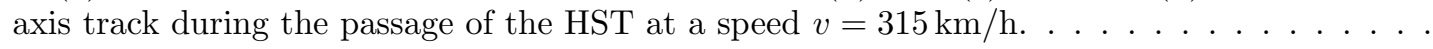

14 The experimental (black line) and computed (grey line) frequency content of the vertical velocity at (a) the sleeper and the free field at a distance of: (b) $4 \mathrm{~m}$; (c) $6 \mathrm{~m}$; and (d) $8 \mathrm{~m}$ from the axis track during the passage of the HST at a speed $v=315 \mathrm{~km} / \mathrm{h}$. . . . . . . . .

15 The experimental (black line), the computed total response (dark grey line) and the computed quasi-static response (light grey line) one-third octave band spectra of the vertical velocity at (a) the sleeper and the free field at a distance of: (b) $4 \mathrm{~m}$; (c) $6 \mathrm{~m}$; and (d) $8 \mathrm{~m}$ from the axis

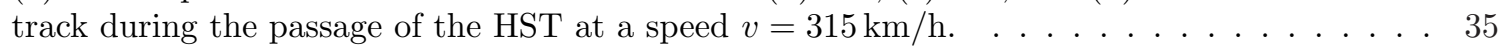

16 (a) Ballasted track system and (b) slab track system. . . . . . . . . . . . . . . . . . 36

17 Transition zone model: (a) Geometry and (b) discretization. . . . . . . . . . . . . . . . . . 37

18 (a-c) Running RMS value and (d-f) one-third octave band spectra of the vertical velocity at $(\mathrm{a}, \mathrm{d})$ the rail, $(\mathrm{b}, \mathrm{e})$ the sleeper, and $(\mathrm{c}, \mathrm{f})$ at a point in the free field located at $11.8 \mathrm{~m}$ from track axis for the stiff rail pad (black line), the medium rail pad (dark grey line), and the soft rail pad (light grey line), at a train speed $v=298 \mathrm{~km} / \mathrm{h}$ travelling on section D. . . . . . . . .

19 Running RMS value of the vertical velocity $(\mathrm{a}, \mathrm{d})$ at the rail, (b,e) the sleeper, and $(\mathrm{c}, \mathrm{f})$ at a point in the free field located at $11.8 \mathrm{~m}$ from track axis for the stiff soil (black line), the medium soil (dark grey line), and the soft soil (light grey line), at a train speed $v=298 \mathrm{~km} / \mathrm{h}$ travelling on: (a-c) section $\mathrm{A}$ and $(\mathrm{d}-\mathrm{f})$ section $\mathrm{D} \ldots \ldots \ldots \ldots \ldots$ 
20 One-third octave band spectra of the vertical velocity (a,d) at the rail, (b,e) the sleeper, and $(\mathrm{c}, \mathrm{f})$ at a point in the free field located at $11.8 \mathrm{~m}$ from track axis for the stiff soil (black line), the medium soil (dark grey line), and the soft soil (light grey line), at a train speed

$21 \quad v=298 \mathrm{~km} / \mathrm{h}$ travelling on: $(\mathrm{a}-\mathrm{c})$ section $\mathrm{A}$ and $(\mathrm{d}-\mathrm{f})$ section $\mathrm{D} \ldots \ldots \ldots \ldots \ldots \ldots$ point in the free field located at $11.8 \mathrm{~m}$ from track axis for the medium soil and the medium rail pad computed from the transition zone model (figure 17) (black line) and from an invariant geometry model (grey line) at a train speed $v=298 \mathrm{~km} / \mathrm{h}$ travelling on: (a,e,i) section A, $(\mathrm{b}, \mathrm{f}, \mathrm{j})$ section $\mathrm{B},(\mathrm{c}, \mathrm{g}, \mathrm{k})$ section $\mathrm{C}$, and $(\mathrm{d}, \mathrm{h}, \mathrm{l})$ section $\mathrm{D} \ldots \ldots \ldots \ldots$. . . . . . . . . 41 


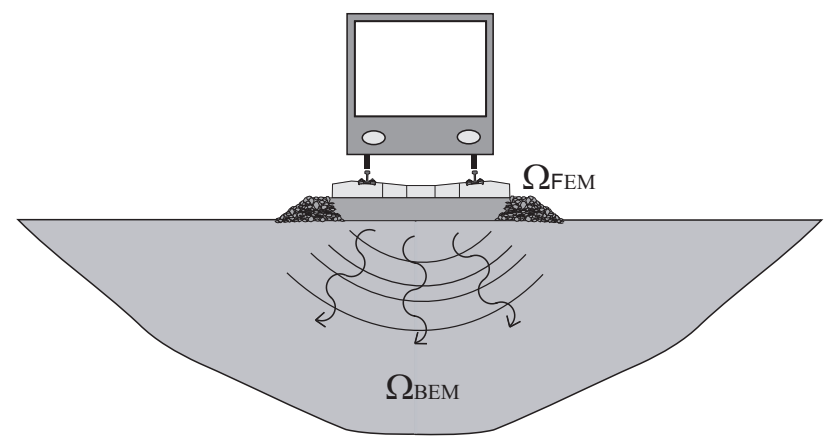

Figure 1: The dynamic track-soil and soil-structure interaction problem. 

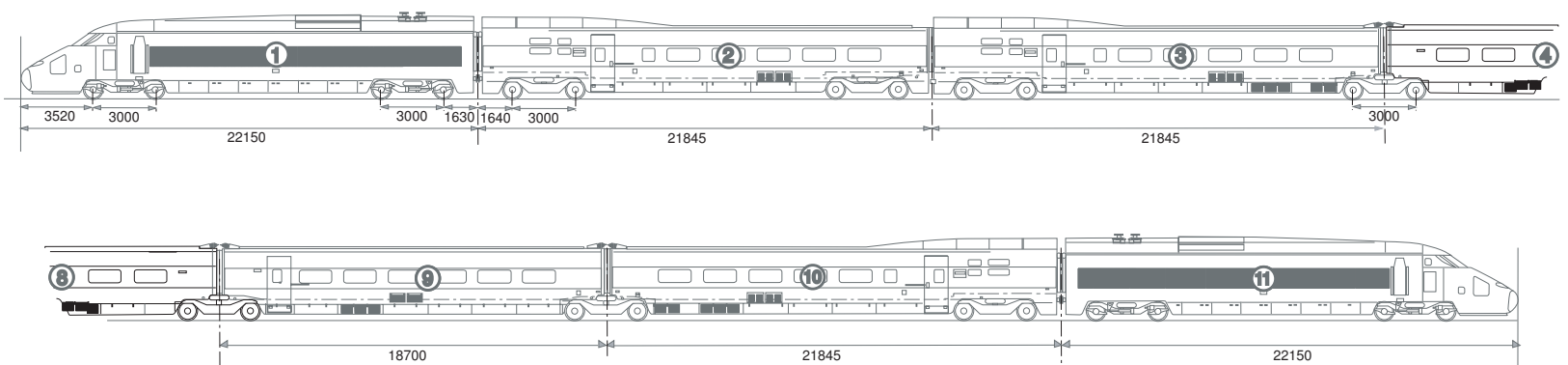

Figure 2: Configuration of the Córdoba-Málaga HST. 


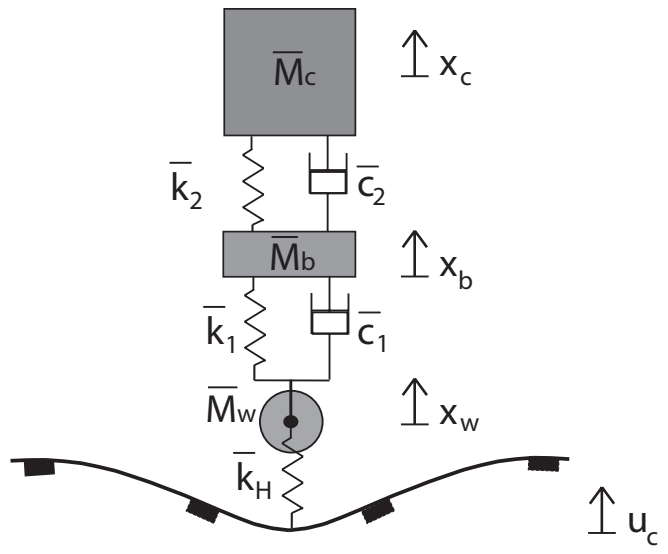

Figure 3: The multi-body model for an axle. 


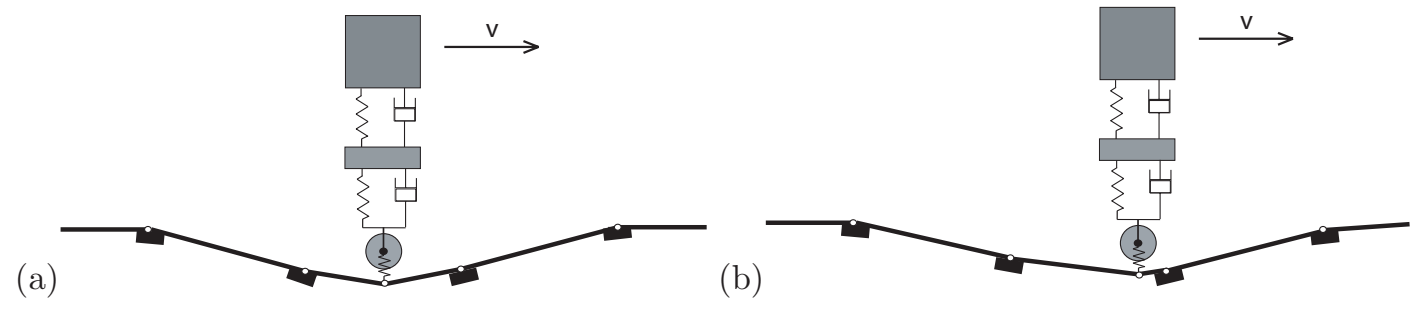

Figure 4: Vehicle and track model at: (a) time step i and (b) time step i+1. 


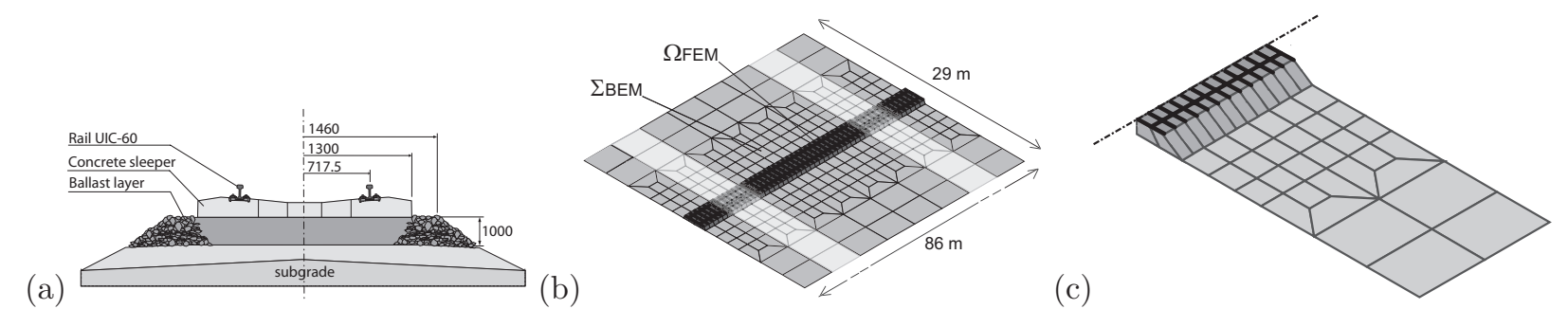

Figure 5: (a) Ballast system track, (b) Track and soil discretization and (c) Detail of the discretization. 
(a)

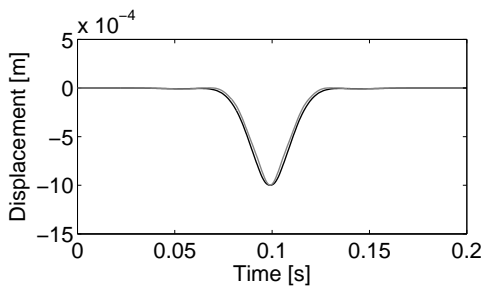

(b)

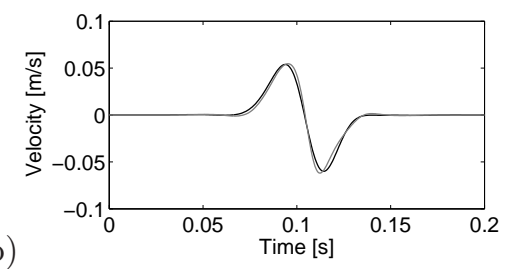

(c)

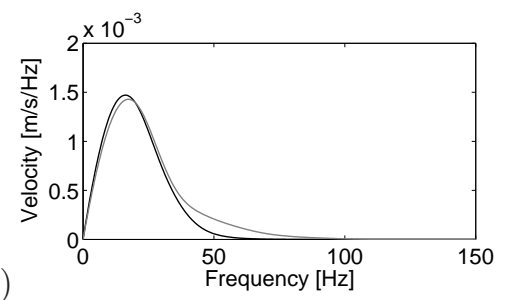

Figure 6: (a) Time history of the vertical displacement and (b) vertical velocity and (c) frequency content of the vertical velocity at the rail for a single axle travelling at $v=298 \mathrm{~km} / \mathrm{h}$ computed from the moving force model (black line) and the multi-body model (grey line). 


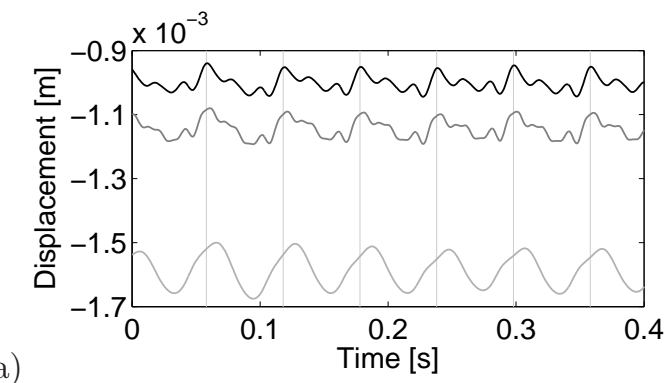

(a)

(c)

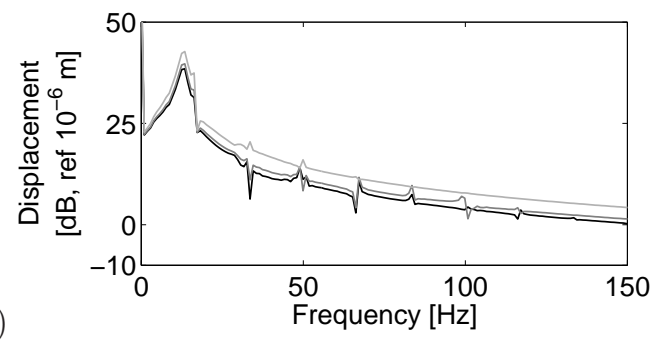

(b)
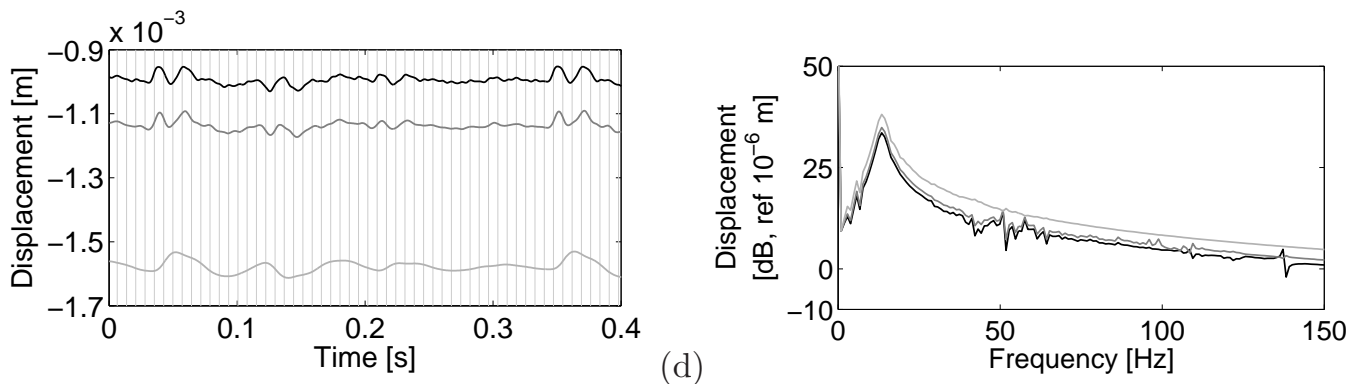

Figure 7: (a,b) Time histories and (c,d) frequency contents of the vertical displacement at the body car (light grey line), bogie (dark grey line) and wheel (black line) due to sleeper discrete support for a single axle travelling at (a,c) $v=36 \mathrm{~km} / \mathrm{h}$ and (b,d) $v=298 \mathrm{~km} / \mathrm{h}$. The sleepers positions are shown as vertical grey lines. 

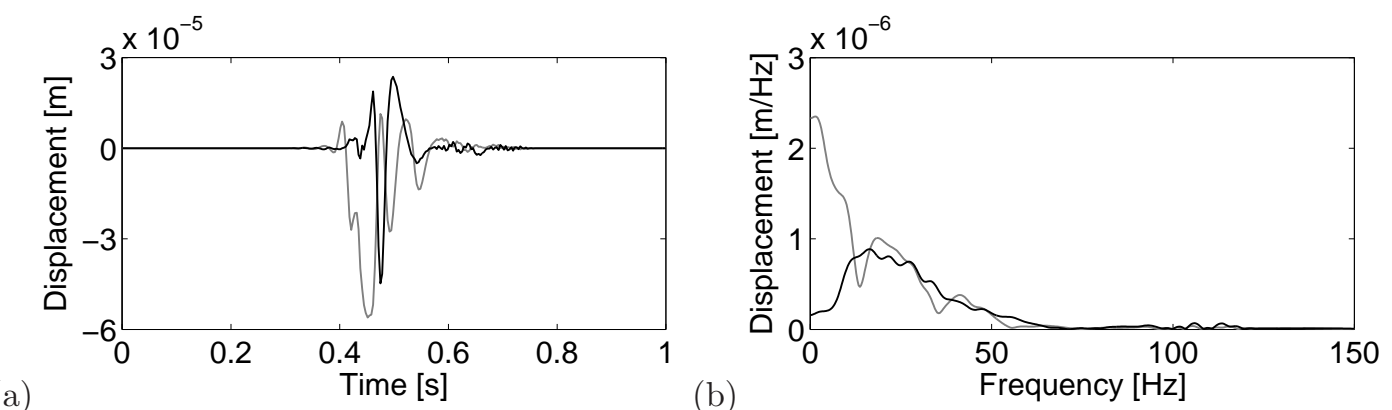

Figure 8: (a) Time history and (b) frequency content of the vertical displacement at the rail for a single axle travelling at $v=298 \mathrm{~km} / \mathrm{h}$ computed from the unsprung mass model (black line) and the multi-body model (grey line). 


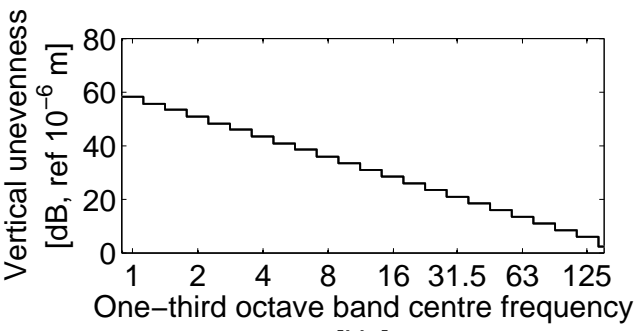

(a)
$[\mathrm{Hz}]$

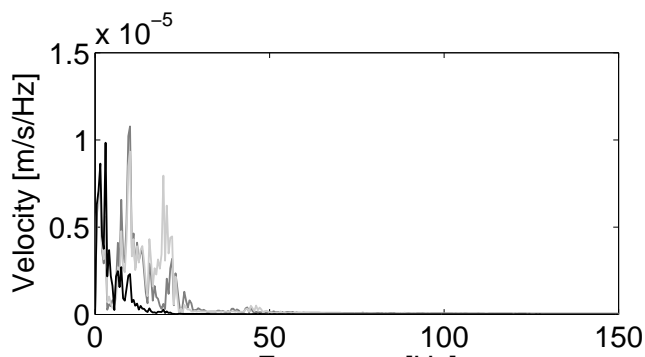

(b)

Frequency [Hz]

Figure 9: (a) One-third octave band spectra of the track unevenness and (b) the frequency content of the vertical velocity at the body car (black line), bogie (light grey line) and wheel (dark grey line) due to unevenness for a single axle travelling at $v=298 \mathrm{~km} / \mathrm{h}$ computed from the multi-body model. 


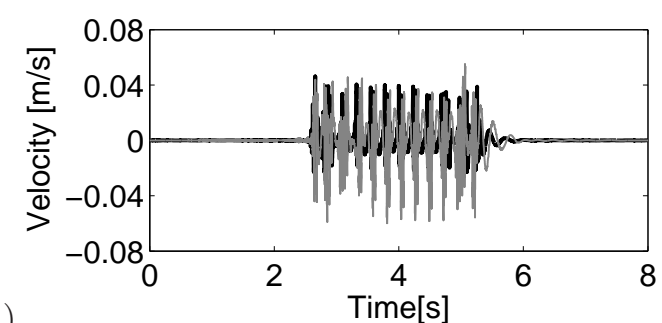

(a)
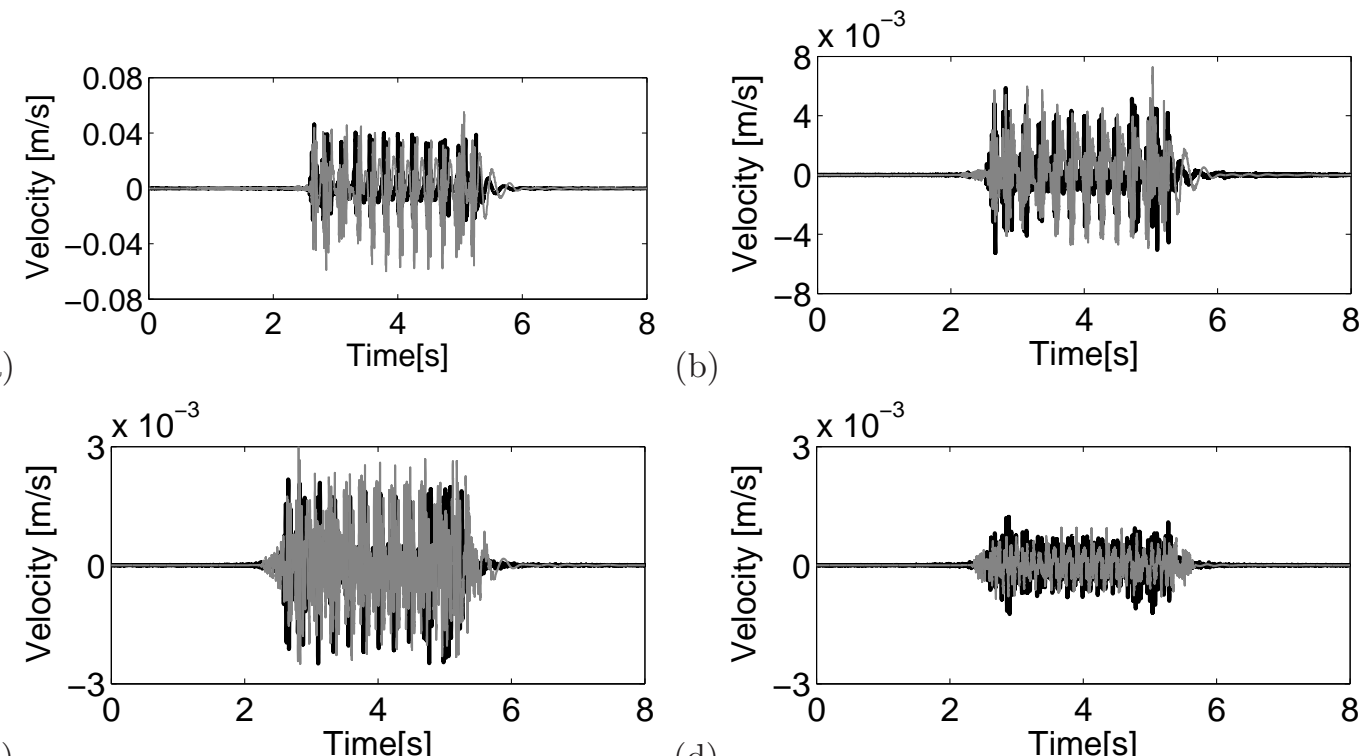

(b)

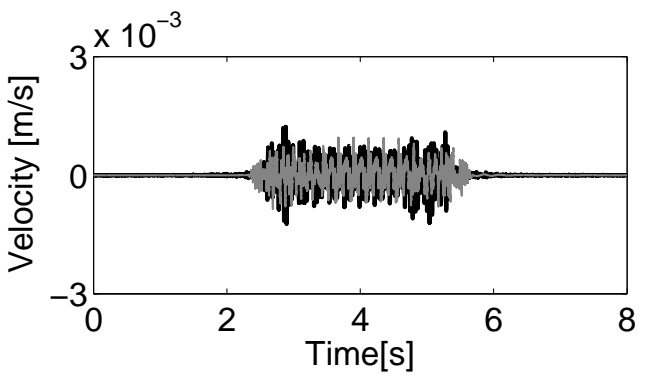

Figure 10: The experimental (black line) and computed (grey line) time history of the vertical velocity at (a) the sleeper and the free field at a distance of: (b) $3 \mathrm{~m}$; (c) $8 \mathrm{~m}$; and (d) $11.8 \mathrm{~m}$ from the axis track during the passage of the HST at a speed $v=298 \mathrm{~km} / \mathrm{h}$. 


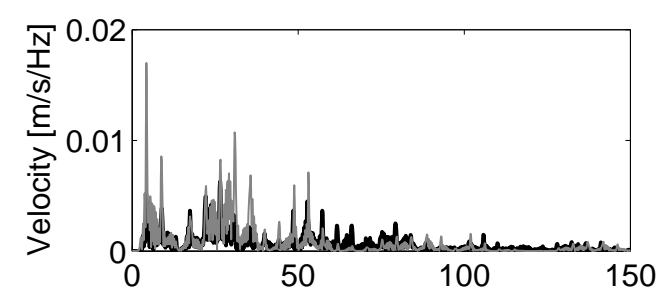

(a)

Frequency $[\mathrm{Hz}]$

(b)
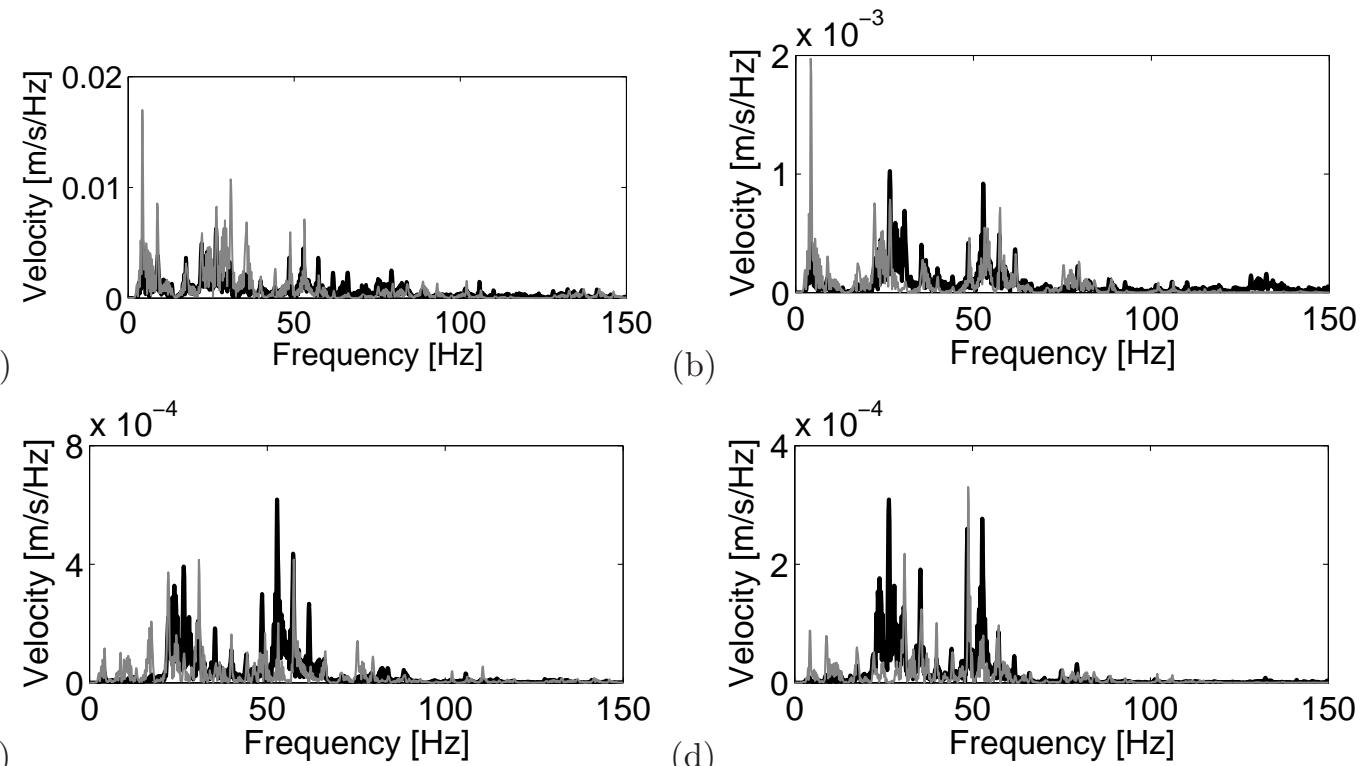

Figure 11: The experimental (black line) and computed (grey line) frequency content of the vertical velocity at (a) the sleeper and the free field at a distance of: (b) $3 \mathrm{~m}$; (c) $8 \mathrm{~m}$; and (d) $11.8 \mathrm{~m}$ from the axis track during the passage of the HST at a speed $v=298 \mathrm{~km} / \mathrm{h}$. 


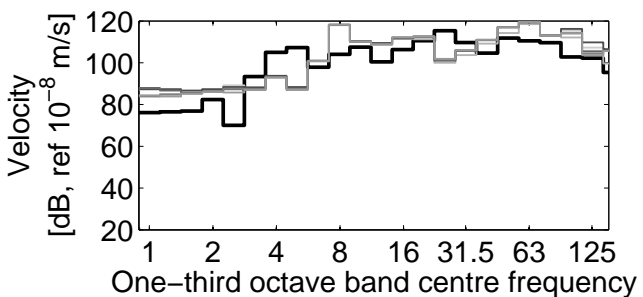

(a) One-third octave band centre frequency

$[\mathrm{Hz}]$

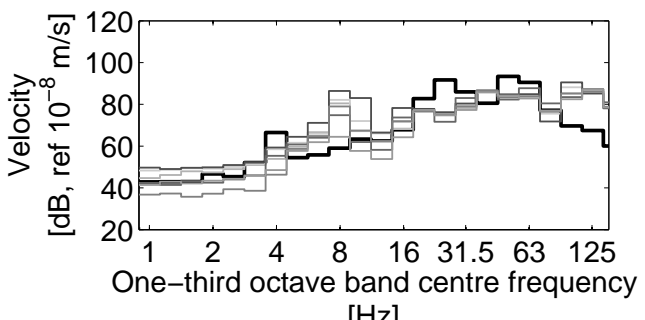

(c)

[Hz]

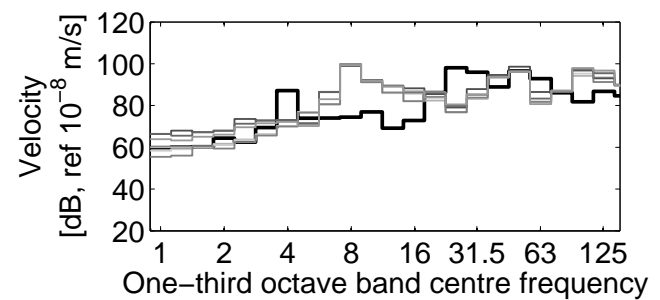

(b)

$[\mathrm{Hz}]$

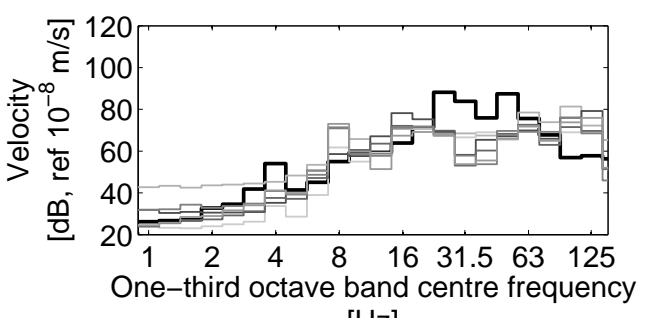

(d)

$[\mathrm{Hz}]$

Figure 12: The experimental (black line) and computed for 6 samples of vertical track unevenness (grey lines) one-third octave band spectra of the vertical velocity at (a) the sleeper and the free field at a distance of: (b) $3 \mathrm{~m}$; (c) $8 \mathrm{~m}$; and (d) $11.8 \mathrm{~m}$ from the axis track during the passage of the HST at a speed $v=298 \mathrm{~km} / \mathrm{h}$. 


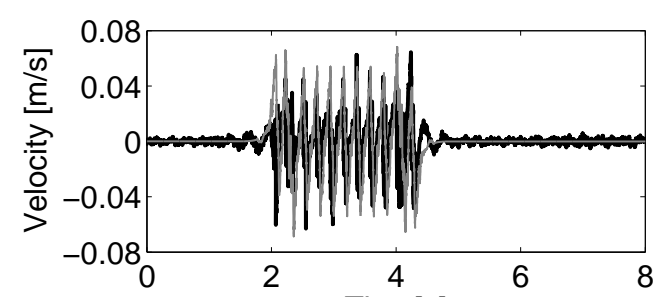

(a)

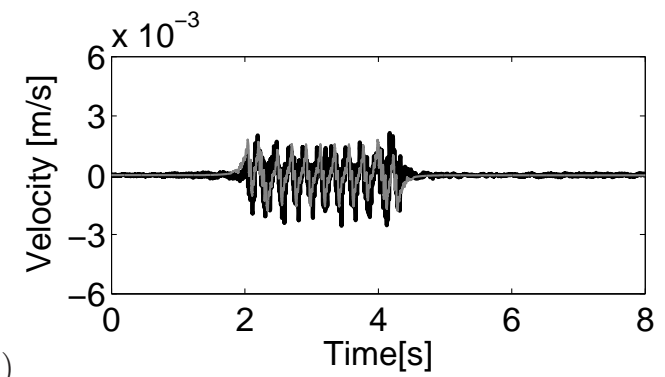

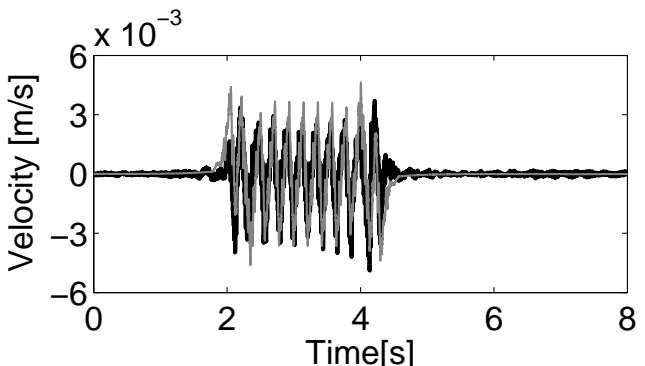

(b)

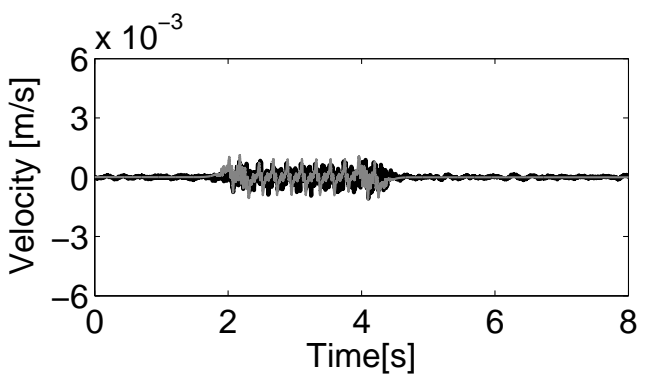

Figure 13: The experimental (black line) and computed (grey line) time history of the vertical velocity at (a) the sleeper and the free field at a distance of: (b) $4 \mathrm{~m}$; (c) $6 \mathrm{~m}$; and (d) $8 \mathrm{~m}$ from the axis track during the passage of the HST at a speed $v=315 \mathrm{~km} / \mathrm{h}$. 


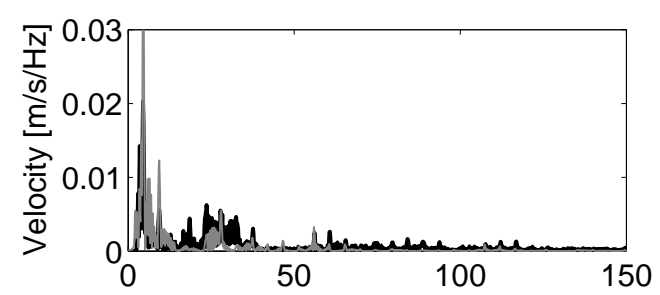

(a)

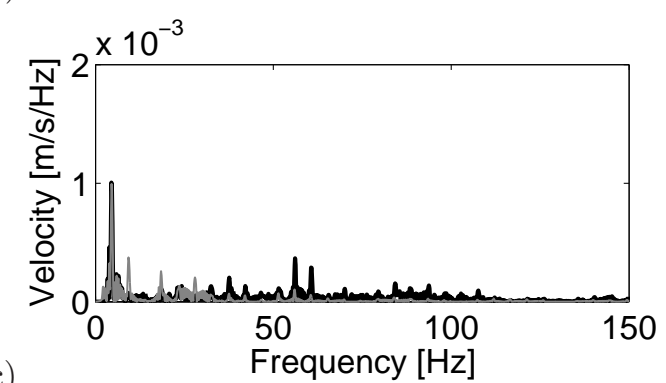

(c)

Frequency $[\mathrm{Hz}]$

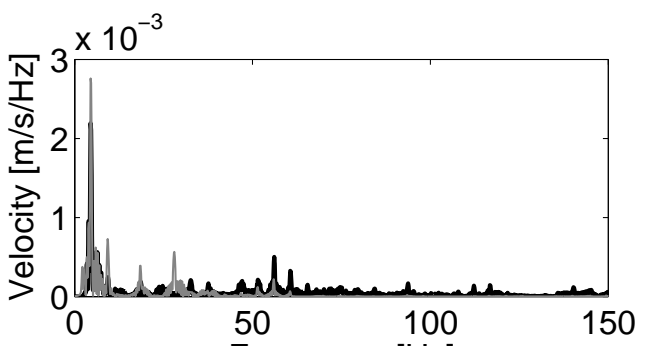

(b)

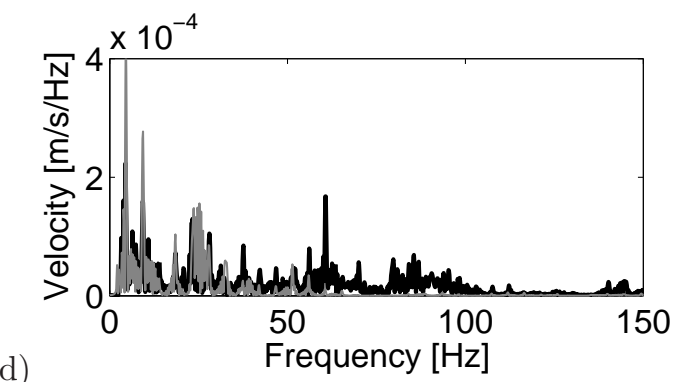

Figure 14: The experimental (black line) and computed (grey line) frequency content of the vertical velocity at (a) the sleeper and the free field at a distance of: (b) $4 \mathrm{~m}$; (c) $6 \mathrm{~m}$; and (d) $8 \mathrm{~m}$ from the axis track during the passage of the HST at a speed $v=315 \mathrm{~km} / \mathrm{h}$. 


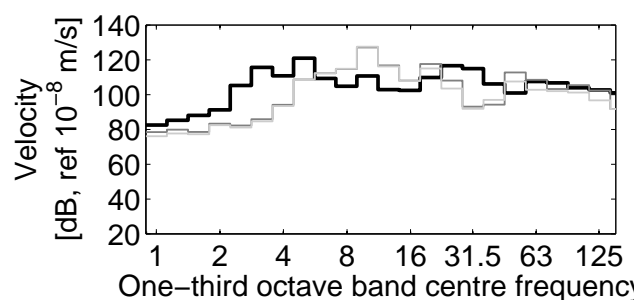

(a) One-third octave band centre frequency

$[\mathrm{Hz}]$

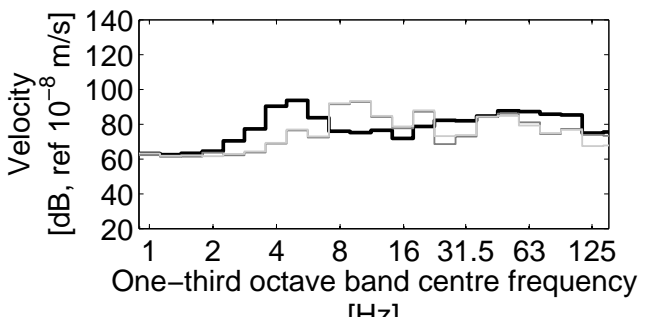

(c)

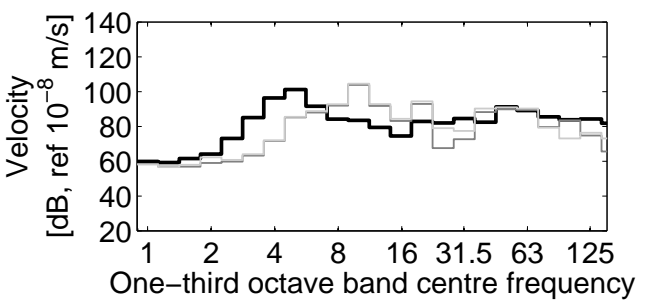

$[\mathrm{Hz}]$

(b)

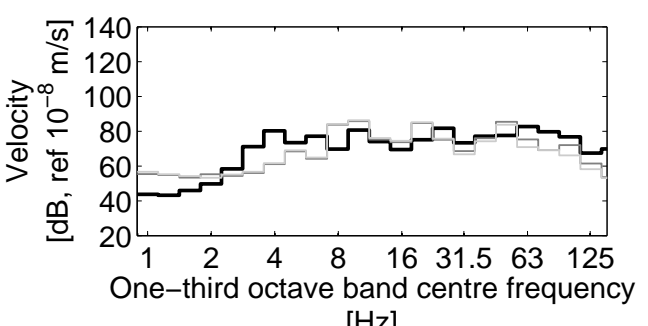

(d)

[Hz]

Figure 15: The experimental (black line), the computed total response (dark grey line) and the computed quasi-static response (light grey line) one-third octave band spectra of the vertical velocity at (a) the sleeper and the free field at a distance of: (b) $4 \mathrm{~m}$; (c) $6 \mathrm{~m}$; and (d) $8 \mathrm{~m}$ from the axis track during the passage of the HST at a speed $v=315 \mathrm{~km} / \mathrm{h}$. 

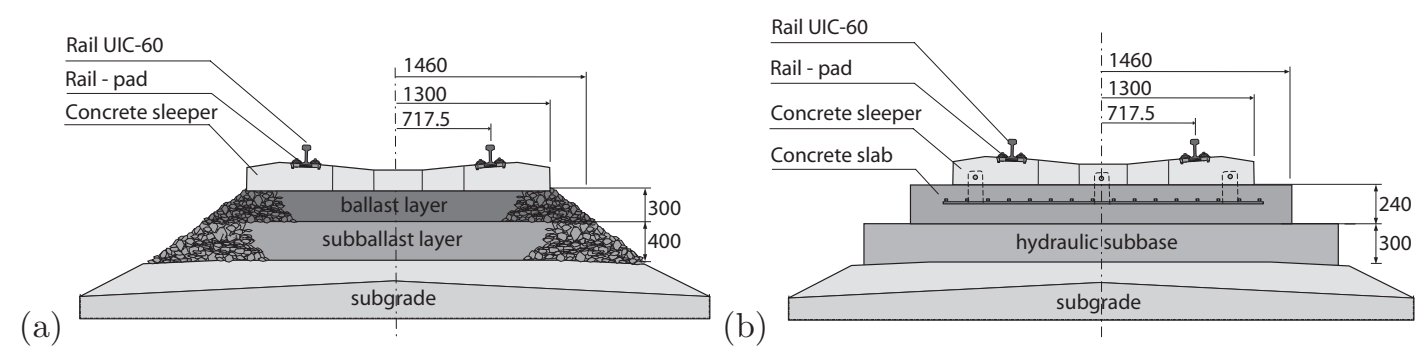

Figure 16: (a) Ballasted track system and (b) slab track system. 

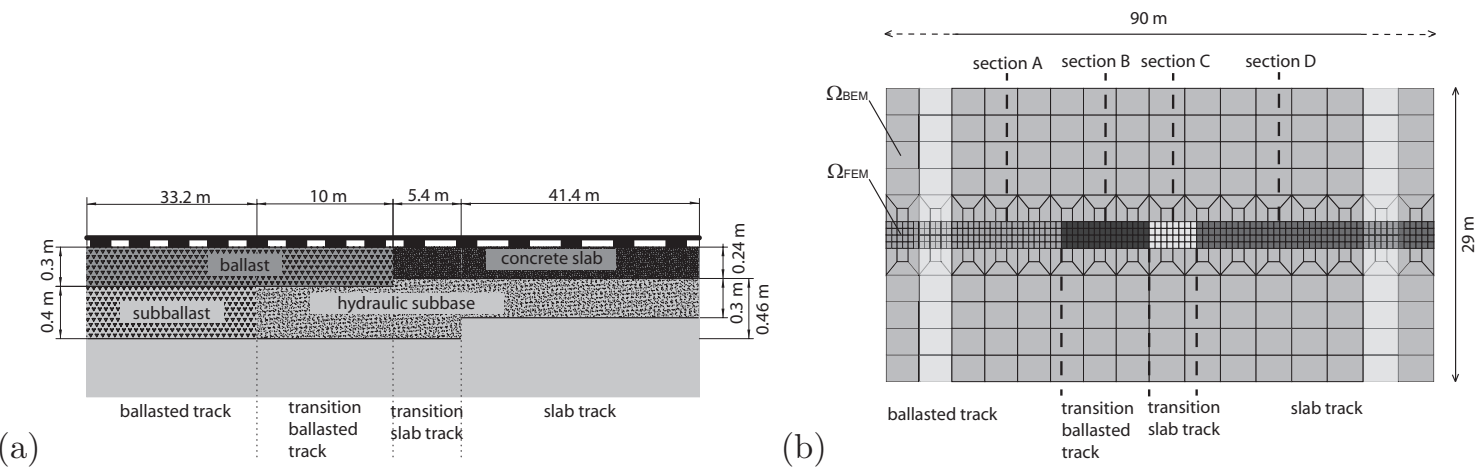

Figure 17: Transition zone model: (a) Geometry and (b) discretization. 
(a)
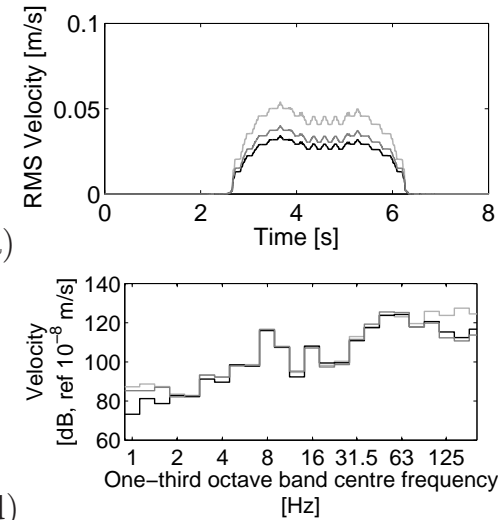

(b)
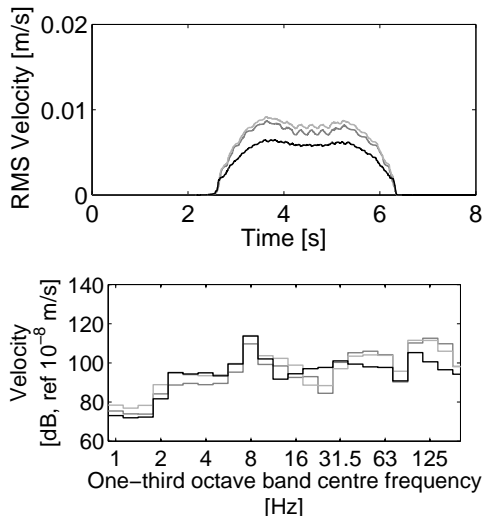

(c)
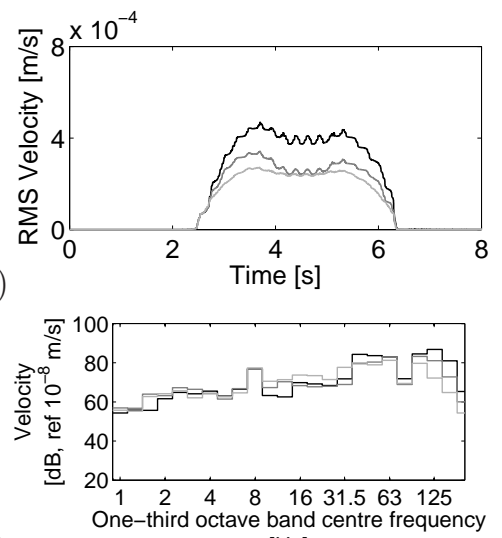

$[\mathrm{Hz}]$

Figure 18: (a-c) Running RMS value and (d-f) one-third octave band spectra of the vertical velocity at (a,d) the rail, (b,e) the sleeper, and $(\mathrm{c}, \mathrm{f})$ at a point in the free field located at $11.8 \mathrm{~m}$ from track axis for the stiff rail pad (black line), the medium rail pad (dark grey line), and the soft rail pad (light grey line), at a train speed $v=298 \mathrm{~km} / \mathrm{h}$ travelling on section D. 
(a)
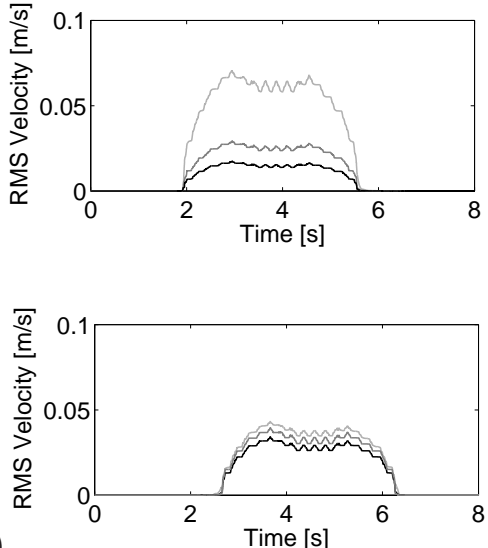

(d)

(b) Time $[\mathrm{s}] \quad$ (c)
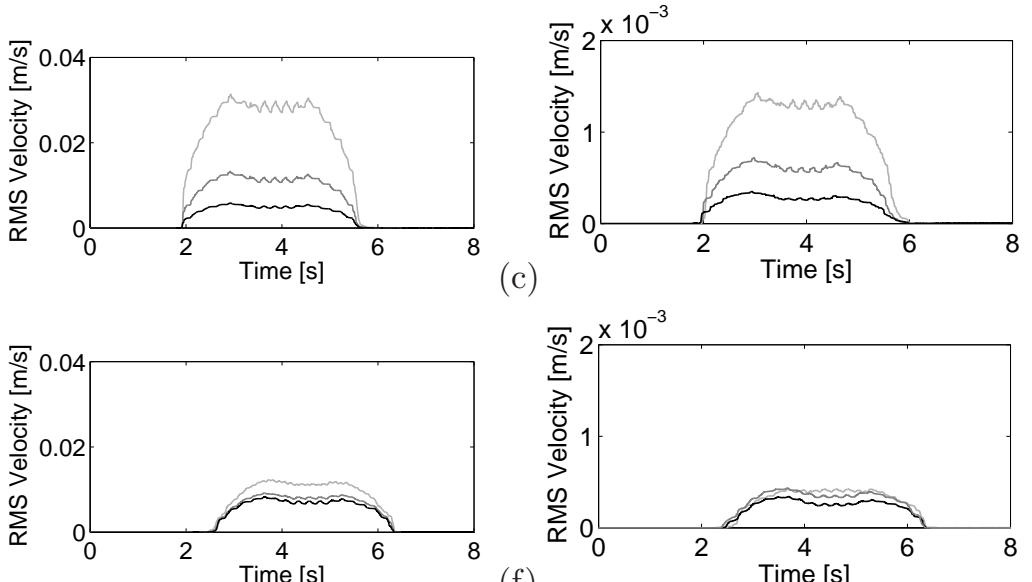

(e)

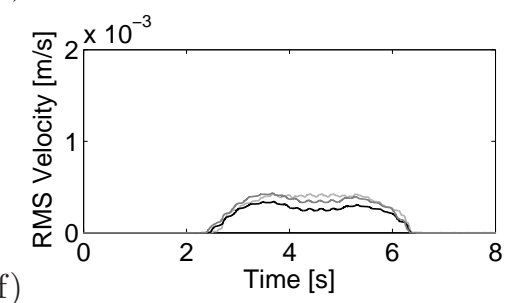

Figure 19: Running RMS value of the vertical velocity (a,d) at the rail, (b,e) the sleeper, and (c,f) at a point in the free field located at $11.8 \mathrm{~m}$ from track axis for the stiff soil (black line), the medium soil (dark grey line), and the soft soil (light grey line), at a train speed $v=298 \mathrm{~km} / \mathrm{h}$ travelling on: (a-c) section A and (d-f) section D. 


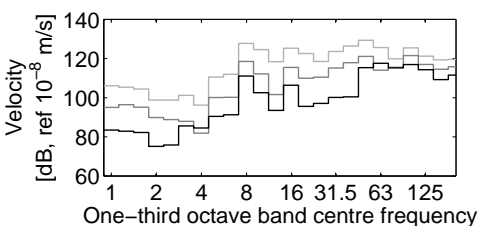

(a)

$[\mathrm{Hz}]$

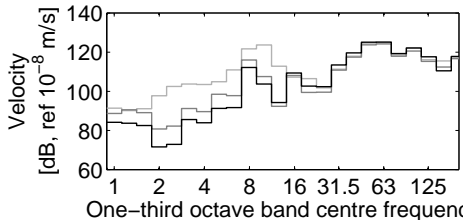

(d)

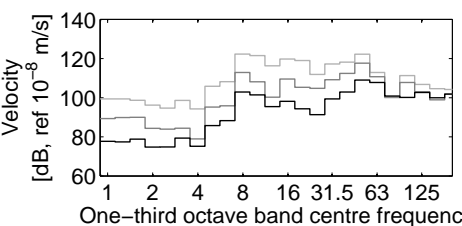

$[\mathrm{Hz}]$

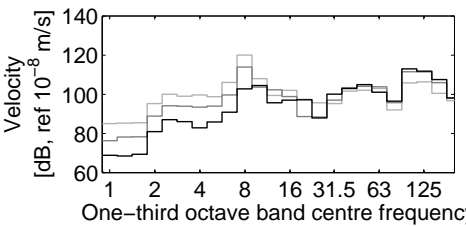

(e)
$[\mathrm{Hz}]$

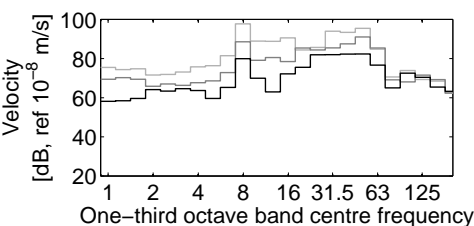

(c)

Figure 20: One-third octave band spectra of the vertical velocity (a,d) at the rail, (b,e) the sleeper, and (c,f) at a point in the free field located at $11.8 \mathrm{~m}$ from track axis for the stiff soil (black line), the medium soil (dark grey line), and the soft soil (light grey line), at a train speed $v=298 \mathrm{~km} / \mathrm{h}$ travelling on: (a-c) section A and (d-f) section D. 
(a)
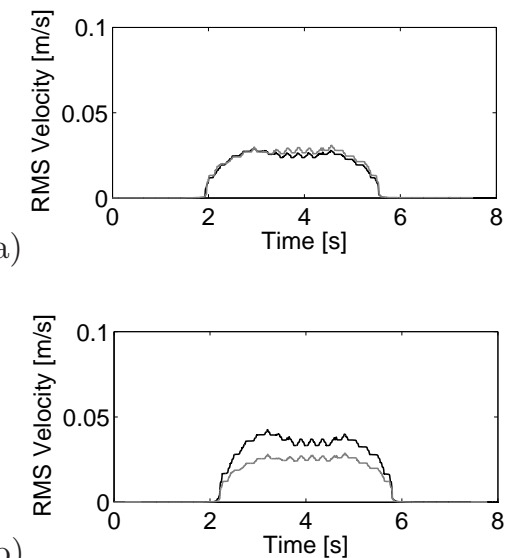

(b)

(c)
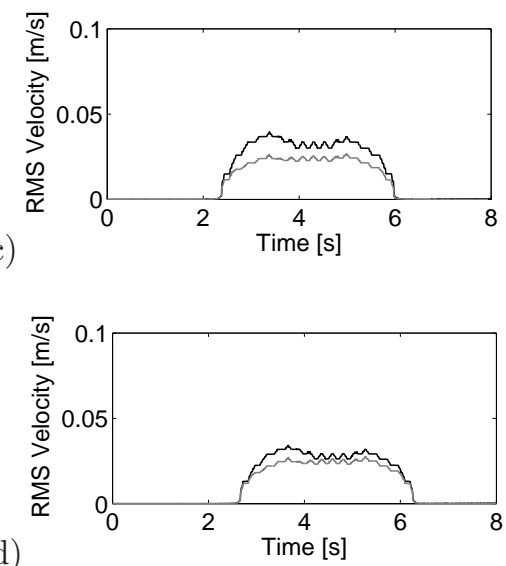

(e)
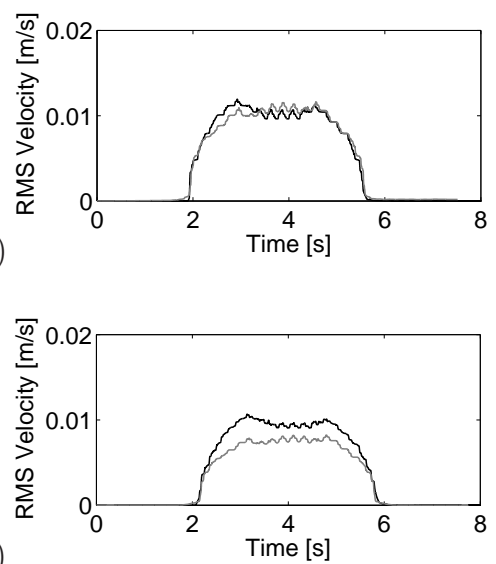

(f)

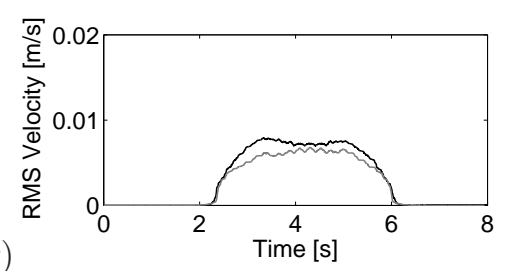

(g)

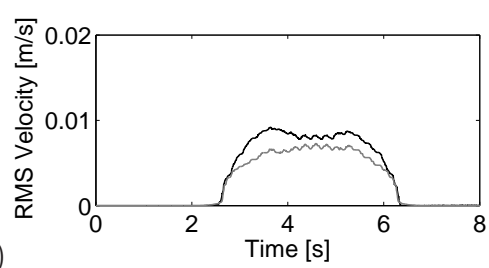

(i)
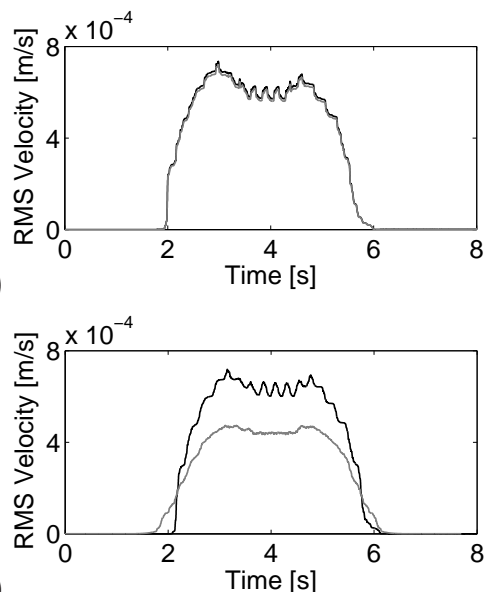

(j)

(k)
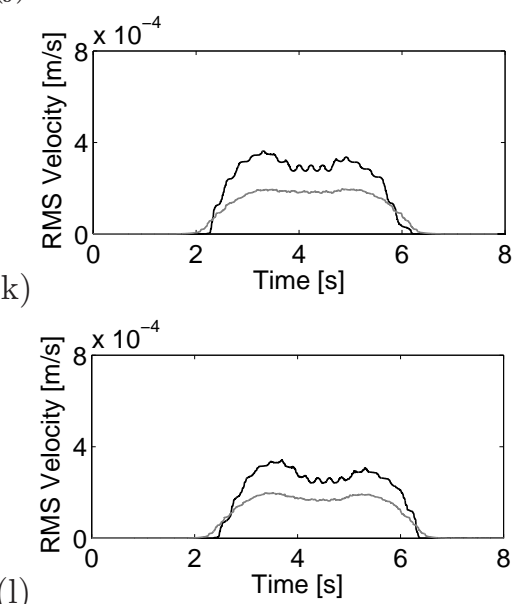

Figure 21: Running RMS value of the vertical velocity (a-d) at the rail, (e-h) the sleeper, and (i-l) at a point in the free field located at $11.8 \mathrm{~m}$ from track axis for the medium soil and the medium rail pad computed from the transition zone model (figure 17) (black line) and from an invariant geometry model (grey line) at a train speed $v=298 \mathrm{~km} / \mathrm{h}$ travelling on: (a,e,i) section A, $(b, f, j)$ section $B,(c, g, k)$ section $C$, and $(d, h, l)$ section D. 


\section{List of Tables}

Geometrical and mass characteristics of the HST . . . . . . . . . . . . . . . . . . . . . . 43

Dynamic characteristics of the primary and secondary suspension of the HST. . . . . . . . . . 44

Rail pad properties. . . . . . . . . . . . . . . . . . . . . . 45

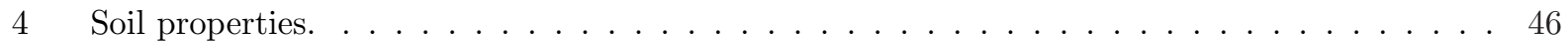




\begin{tabular}{cccccccc}
\hline & No. of carriages & No. of axles & $L_{t}[\mathrm{~m}]$ & $L_{b}[\mathrm{~m}]$ & $L_{a}[\mathrm{~m}]$ & $M_{u}[\mathrm{~kg}]$ & $M_{t}[\mathrm{~kg}]$ \\
\hline Traction cars & 2 & 4 & 22.15 & 14.00 & 3.00 & 2048 & 17185 \\
End carriages & 2 & 3 & 21.84 & 18.70 & 3.00 & 2003 & 11523 \\
Central carriages & 6 & 2 & 18.70 & 18.70 & 3.00 & 2003 & 15523 \\
\hline
\end{tabular}

Table 1: Geometrical and mass characteristics of the HST. 


\begin{tabular}{ccccc}
\hline & $k_{1}$ & $c_{1}$ & $k_{2}$ & $c_{2}$ \\
& $\times 10^{6}[\mathrm{~N} / \mathrm{m}]$ & $\times 10^{3}[\mathrm{Ns} / \mathrm{m}]$ & $\times 10^{6}[\mathrm{~N} / \mathrm{m}]$ & $\times 10^{3}[\mathrm{Ns} / \mathrm{m}]$ \\
\hline Traction cars & 1.2 & 10 & 2.45 & 40 \\
Passenger car & 0.7 & 5 & 0.82 & 48 \\
\hline
\end{tabular}

Table 2: Dynamic characteristics of the primary and secondary suspension of the HST. 


\begin{tabular}{ccc}
\hline Rail pad type & $\begin{array}{c}k_{r p} \\
\times 10^{6}[\mathrm{~N} / \mathrm{m}]\end{array}$ & $\begin{array}{c}c_{r p} \\
\times 10^{3}[\mathrm{Ns} / \mathrm{m}]\end{array}$ \\
\hline Stiff & 360 & 13.5 \\
Medium & 150 & 13.5 \\
Soft & 60 & 13.5 \\
\hline
\end{tabular}

Table 3: Rail pad properties. 


\begin{tabular}{cccc}
\hline Soil type & $C_{s}[\mathrm{~m} / \mathrm{s}]$ & $C_{p}[\mathrm{~m} / \mathrm{s}]$ & $C_{R}[\mathrm{~m} / \mathrm{s}]$ \\
\hline Stiff & 250.0 & 499.6 & 232.9 \\
Medium & 150.0 & 299.8 & 139.7 \\
Soft & 80.0 & 150.0 & 74.1 \\
\hline
\end{tabular}

Table 4: Soil properties. 